\title{
How does it scale? Comparing quantum and classical nonlinear optical processes in integrated devices
}

\author{
Lukas G. Helt, ${ }^{1, *}$ Marco Liscidini, ${ }^{2}$ and John E. Sipe ${ }^{1}$ \\ ${ }^{1}$ Department of Physics, University of Toronto, 60 St. George Street, Toronto, Ontario M5S 1A7, Canada \\ ${ }^{2}$ Dipartimento di Fisica, Università delgi studi di Pavia, via Bassi 6, I-27100 Pavia, Italy \\ ${ }^{*}$ Corresponding author: Ihelt@physics.utoronto.ca
}

Received May 1, 2012; accepted June 5, 2012;

posted June 13, 2012 (Doc. ID 167658); published August 1, 2012

\begin{abstract}
We present equations for the power generated via spontaneous (quantum) and stimulated (classical) nonlinear optical processes in integrated devices. Equations for the same structure and same order process are derived from the same Hamiltonian, allowing for direct and easy comparison including the ability to estimate the efficiency of a quantum process based solely on experimental data from a classical process in the same device. We show that, in the CW limit and under the undepleted pump approximation, the average energy of a generated photon divided by a characteristic time plays the role of the classical "seed" signal in a quantum process, and that extending the length of a structure or taking advantage of a resonant cavity does not enhance spontaneous processes the same way as stimulated processes. (C) 2012 Optical Society of America
\end{abstract} OCIS codes: $\quad 190.4380,190.4390,190.4410,270.0270$.

\section{INTRODUCTION}

The field of nonlinear optics, first explored more than 50 years ago [1], continues today to see improvements in conversion efficiency and reductions in system size driven by a steady progression from bulk-crystal optics to integrated waveguides. Although integrated devices can require more sophisticated fabrication techniques, they allow for tighter modal confinement and better modal overlap than bulk crystals, leading to higher conversion and collection efficiencies, not to mention better scalability and integration with existing infrastructure. While often designed with the enhancement of a classical nonlinear optical process in mind, these integrated devices will also enhance the corresponding quantum nonlinear optical process. Thus many structures exist today that could potentially be used for the generation of quantum correlated photon pairs (see, e.g., [2]).

But just how efficient will this photon pair generation be? Do enhancements to the performance of devices in classical experiments scale the same way as in photon pair generation experiments? How does one estimate the efficiency of photon pair generation in a specific device given the results of a classical experiment? Here we aim at answering these questions utilizing a general Hamiltonian formalism that places quantum wave mixing processes, such as spontaneous parametric downconversion (SPDC) or spontaneous four-wave mixing (SFWM), and classical wave mixing processes, such as difference frequency generation (DFG) or classical four-wave mixing (FWM), on equal theoretical footing. This approach makes it easy to compare how each process scales, and identify terms common to quantum and classical processes. The formalism can be applied to integrated nonlinear structures, such as channel waveguides or microring resonators side-coupled to channel waveguides, when either a second- or third-order nonlinearity is dominant, and takes into account both material and modal dispersion. While losses, quantum fluctuations in stimulated experiments, generated photons seeding stimulated processes in spontaneous experiments, and nonlinear effects such as self- and cross-phase modulation can be important in integrated devices, we focus here on developing simple and intuitive scaling relationships, and so defer their inclusion to later publications. In what follows, we neglect all forms of loss as well as all nonlinear effects other than the process at hand, and present results only strictly valid in the undepleted pump approximation.

Note that when we write corresponding processes, we do not mean reverse processes. For example, SPDC is a quantum $\chi_{2}$ process in which a pump photon at $\omega_{P}$ is converted into a photon at each $\omega_{S}$ and $\omega_{I}$, with $\omega_{P}=\omega_{S}+\omega_{I}$ (see Fig. 1). The reverse process is second harmonic generation ( $\mathrm{SHG}$ ), in which two pump photons at $\omega_{F}$ are converted into a photon at $\omega_{\mathrm{SH}}$ with $2 \omega_{F}=\omega_{\mathrm{SH}}$ or, more properly, sum frequency generation (SFG), in which pump photons at each $\omega_{S}$ and $\omega_{I}$ are converted into a photon at $\omega_{P}$. And so it might seem natural to seek a correspondence between the efficiencies of SPDC and SFG. Yet, while it is true that knowledge of the efficiency of either SHG or SFG in a given device- or indeed that of any second-order nonlinear optical process-will allow one to infer something about the efficiency of SPDC in the same device, we find it most convenient to compare quantum and classical processes each pumped at the same frequency. Therefore we refer to DFG, in which a pump photon at $\omega_{P}$ as well as a seed photon at $\omega_{S}$ create a photon at $\omega_{I}$, as the classical $\chi_{2}$ process corresponding to SPDC (see Fig. 2). Similarly the classical $\chi_{3}$ process corresponding to SFWM, where two pump photons at $\omega_{P}$ are converted into a photon at each $\omega_{S}$ and $\omega_{I}$, with $2 \omega_{P}=\omega_{S}+\omega_{I}$ (see Fig. 1), is FWM, where two pump photons at $\omega_{P}$ as well as a seed photon at $\omega_{S}$ lead to a photon at $\omega_{I}$. The $\chi_{3}$ process that is the reverse of SFWM is dual-pump SFWM, in which photons at each $\omega_{S}$ and $\omega_{I}$ are converted into two photons at $\omega_{P}$. Classical processes 


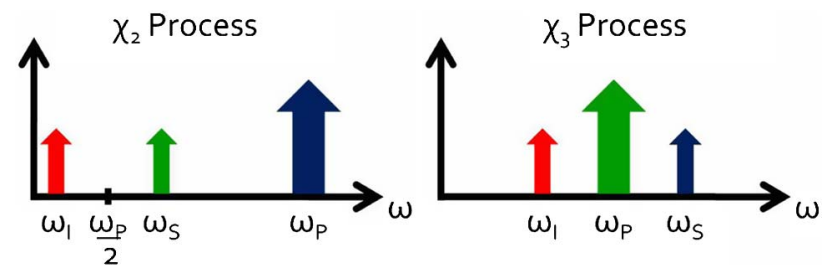

Fig. 1. (Color online) Schematic of frequency spacings of secondand third-order nonlinear optical processes.

corresponding to our spontaneous quantum processes of interest are thus their stimulated counterparts.

The remainder of the paper is organized as follows. In Section 2, we present and compare formulae derived for corresponding quantum and classical efficiencies for both $\chi_{2}$ and $\chi_{3}$ processes in channel waveguides. In Section 3 , we do the same for a resonant structure, choosing a microring resonator side-coupled to a single channel waveguide as an example. Despite the fact that the physics of corresponding spontaneous and stimulated processes is most easily related, it is also generally true that it is experimentally easier to determine the efficiency of SHG than that of DFG. So as an aid in estimating the effectiveness of optical devices as SPDC sources based on the results of classical experiments, we also present a short discussion of SHG efficiency within our general framework. In Section IV, we conclude. The details of the quantum and classical calculations for a $\chi_{3}$ process in a channel waveguide are presented in Appendices A and B, respectively, to serve as an example and model of the form of our calculations.

\section{NONRESONANT CASE}

Here we consider spontaneous processes and their corresponding stimulated processes in a device such as a channel waveguide [3-5], nonlinear fiber [6,7], or Bragg reflection waveguide [8], where propagation can be treated as effectively one-dimensional. We first consider $\chi_{2}$ processes.

\section{A. Second-Order Processes}

SPDC in such a structure was considered in an earlier work, and so in this section we only summarize results, directing the reader to [9] for details. As in that work, we take our Hamiltonian to be

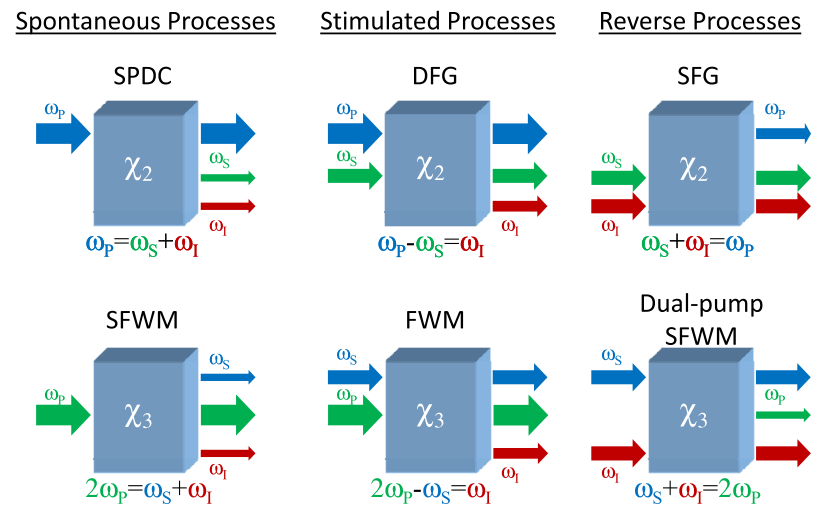

Fig. 2. (Color online) Schematic of frequencies generated by secondand third-order nonlinear optical processes, organized according to spontaneous, stimulated, and reverse processes, with corresponding processes occupying the first two columns.

$$
\begin{gathered}
H=H_{L}+H_{N L}, \\
H_{L}=\int \mathrm{d} k \hbar \omega_{F k} a_{F k}^{\dagger} a_{F k}+\int \mathrm{d} k \hbar \omega_{\mathrm{SH} k} a_{\mathrm{SH} k}^{\dagger} a_{\mathrm{SH} k},
\end{gathered}
$$

$$
H_{N L}=-\int \mathrm{d} k_{1} \mathrm{~d} k_{2} \mathrm{~d} k S\left(k_{1}, k_{2}, k\right) a_{F k_{1}}^{\dagger} a_{F k_{2}}^{\dagger} a_{\mathrm{SH} k}+\text { H.c. }
$$

where the $\omega_{m k}$ are eigenfrequencies of the photon modes (e.g., lowest-order TE-like mode) labeled by $m=F(\mathrm{SH})$ for the fundamental (second harmonic) frequency region following the terminology of SHG (see Fig. 1), and $S\left(k_{1}, k_{2}, k\right)$ is a nonlinear coupling coefficient defined previously [9]. Note that while here, for simplicity, we have implicitly assumed that both fundamental fields have the same polarization (as in a Type-I process), generalizations are straightforward [10].

Following the approach outlined in [9], the state of SPDC generated photons in a channel waveguide due to an exciting pump pulse is written as the two-mode squeezed vacuum

$$
\left|\psi_{\text {gen }}\right\rangle=\exp \left(\beta C_{\mathrm{II}}^{\dagger}-\text { H.c. }\right)|\mathrm{vac}\rangle,
$$

where $|\beta|^{2}$ is proportional to the average number of photons in the pump pulse $\mathcal{N}_{P}$ and, switching to a description in terms of frequency rather than wavenumber [9],

$$
C_{\mathrm{II}}^{\dagger}=\frac{1}{\sqrt{2}} \int \mathrm{d} \omega_{1} \mathrm{~d} \omega_{2} \phi\left(\omega_{1}, \omega_{2}\right) a_{F \omega_{1}}^{\dagger} a_{F \omega_{2}}^{\dagger},
$$

such that $C_{\mathrm{II}}^{\dagger}|\mathrm{vac}\rangle$ is a normalized two-photon state characterized by the biphoton wave function

$$
\begin{aligned}
\phi\left(\omega_{1}, \omega_{2}\right)= & i L \sqrt{\frac{d k_{F}\left(\omega_{1}\right)}{d \omega_{1}}} \sqrt{\frac{d k_{F}\left(\omega_{2}\right)}{d \omega_{2}}}\left(\sqrt{\frac{d k_{\mathrm{SH}}(\omega)}{d \omega}}\right)_{\omega=\omega_{1}+\omega_{2}} \\
& \times \sqrt{\frac{\omega_{1} \omega_{2}\left(\bar{\chi}_{2}\right)^{2} \mathcal{N}_{P}}{8 \pi \varepsilon_{0}|\beta|^{2} \bar{n}^{6}}} \sqrt{\frac{\hbar\left(\omega_{1}+\omega_{2}\right)}{\mathcal{A}\left[k_{F}\left(\omega_{1}\right), k_{F}\left(\omega_{2}\right), k_{\mathrm{SH}}\left(\omega_{1}+\omega_{2}\right)\right]}} \\
& \times \phi_{P}\left(\omega_{1}+\omega_{2}\right) \operatorname{sinc}\left\{\left[k_{F}\left(\omega_{1}\right)+k_{F}\left(\omega_{2}\right)\right.\right. \\
& \left.\left.-k_{\mathrm{SH}}\left(\omega_{1}+\omega_{2}\right)\right] L / 2\right\} .
\end{aligned}
$$

Here $L$ is the length of the nonlinear structure, $\phi_{P}(\omega)$ is the pump pulse waveform, $\bar{n}$ and $\bar{\chi}_{2}$ are, respectively, a typical value of refractive index and a typical value of second-order nonlinearity introduced solely for convenience, and the $k_{m}(\omega)$ are frequency-dependent wavenumbers defined such that the structure is phase matched when $2 k_{F}\left(\omega_{F_{0}}\right)-k_{\mathrm{SH}}\left(\omega_{\mathrm{SH}_{0}}\right)=0$. Furthermore

$$
\begin{aligned}
& \mathcal{A}\left[k_{F}\left(\omega_{1}\right), k_{F}\left(\omega_{2}\right), k_{\mathrm{SH}}\left(\omega_{1}+\omega_{2}\right)\right] \\
& =\left|\int \mathrm{d} x \mathrm{~d} y \frac{\bar{n}^{3} \chi_{2}^{i j k}(x, y)\left[d_{F \omega_{1}}^{i}(x, y)\right]^{*}\left[d_{F \omega_{2}}^{j}(x, y)\right]^{*} d_{\mathrm{SH}\left(\omega_{1}+\omega_{2}\right)}^{k}(x, y)}{\bar{\chi}_{2} \varepsilon_{0}^{3 / 2} n^{2}\left(x, y ; \omega_{1}\right) n^{2}\left(x, y ; \omega_{2}\right) n^{2}\left(x, y ; \omega_{1}+\omega_{2}\right)}\right|^{-2}
\end{aligned}
$$

is an effective area with $\chi_{2}^{i j k}(x, y)$ the material second-order optical nonlinearity neglecting dispersion and magneto-optic effects, $d_{m \omega}^{i}(x, y)$ the $i$ th component of the displacement 
field for mode $m$ at frequency $\omega$, and $n(x, y ; \omega)$ the material refractive index at frequency $\omega$, all at waveguide crosssectional position $(x, y)$, and we have chosen the mode amplitudes such that we can take the phase associated with the effective area to be zero [9]. That $C_{\mathrm{II}}^{\dagger}|\mathrm{vac}\rangle$ is normalized requires

$$
\int \mathrm{d} \omega_{1} \mathrm{~d} \omega_{2}\left|\phi\left(\omega_{1}, \omega_{2}\right)\right|^{2}=1,
$$

which in turn determines $|\beta|$. In the limit $|\beta| \ll 1$,

$$
\left|\psi_{\text {gen }}\right\rangle \approx|\operatorname{vac}\rangle+\beta C_{\mathrm{II}}^{\dagger}|\mathrm{vac}\rangle
$$

and $2|\beta|^{2} \equiv \mathcal{N}_{D}$ can be thought of as the average number of generated photons per pump pulse. Indeed this line of thinking holds even in the limit of a long pulse, with an intensity spectrum that can be approximated as a Dirac delta function, $\left|\phi_{P}\left(\omega_{1}+\omega_{2}\right)\right|^{2} \approx \delta\left(\omega_{1}+\omega_{2}-\omega_{P}\right)$, in comparison to the phasematching sinc function of the biphoton wave function of Eq. (6); the result for $\mathcal{N}_{D}$ is then

$$
\mathcal{N}_{D}=\frac{2 \hbar \omega_{P} \mathcal{N}_{P} L^{2}}{\mathcal{P} \mathcal{A} \mathcal{T}}
$$

provided $|\beta| \ll 1$. Note that in deriving Eq. (10), we have integrated over $\omega_{2}$, and taken the effective area, $\mathcal{A}$, and group velocities, $v_{m}^{-1}=\left(d k_{m} / d \omega\right)_{\omega=\omega_{m_{0}}}$, as constant over the generation bandwidth time explicit time dependence built in so as to simplify the Heisenberg equations of motion

$$
G_{m}(z, t)=\left(\hbar \omega_{m} v_{m} /(2 \pi)\right)^{1 / 2} \int \mathrm{d} k e^{i k z} a_{m k} e^{i\left(\omega_{m} t-k_{m} z\right)},
$$

where the integral goes over the range of $k$ space appropriate for the mode of interest, such that $G_{m}^{\dagger} G_{m}=P_{m}$ has units of power. For a DFG calculation, while the signal and idler may or may not exist in two distinct propagation modes of the waveguide, their center frequencies may be separated enough that we may still define a $G_{m}(z, t)$ for each. Indeed, because of this separation, the points at which the $k$ 's appearing in the $G_{m}(z, t)$ are most naturally expanded about may be well-separated, and as such the ranges over which each $e^{i\left(k-k_{m}\right) z}$ is significantly nonzero will also be well-separated, meaning we can introduce two $m$ labels ( $m=S$ for signal, $m=I$ for idler) corresponding to what was formerly just $F$, with $\left[G_{S}(z, t), G_{I}^{\dagger}(z, t)\right]=0$, and one $m=P$ label for the pump corresponding to what was formerly $\mathrm{SH}$. This is most certainly the case for the $\mathrm{CW}$ process that we consider here. We thus rewrite our initial Hamiltonian (1) as

$$
\begin{aligned}
H^{L}= & \sum_{m=P, S, I} \int \mathrm{d} z G_{m}^{\dagger} G_{m} / v_{m} \\
& +\frac{i}{2 \omega_{m}} \int \mathrm{d} z\left(\frac{\partial G_{m}^{\dagger}}{\partial z} G_{m}-G_{m}^{\dagger} \frac{\partial G_{m}}{\partial z}\right),
\end{aligned}
$$

$$
\mathcal{T}=\frac{2 \pi}{\int_{0}^{\omega_{P} / 2} \mathrm{~d} \Omega\left[1-\left(2 \Omega / \omega_{P}\right)^{2}\right] \operatorname{sinc}^{2}\left\{\left[k_{F}\left(\omega_{P} / 2+\Omega\right)+k_{F}\left(\omega_{P} / 2-\Omega\right)-k_{\mathrm{SH}}\left(\omega_{P}\right)\right] L / 2\right\}},
$$

where $\Omega=\omega_{1}-\omega_{P} / 2$ represents positive detuning from $\omega_{P} / 2$, and $\mathcal{P}=8 \varepsilon_{0} \bar{n}^{6} v_{F}^{2} v_{\mathrm{SH}} /\left(\bar{\chi}_{2} \omega_{P}\right)^{2}$ is a characteristic power for a $\chi_{2}$ material [11].

Yet it is also true that if we consider a constant rate of pump photons or average pump power, $P_{P}=\hbar \omega_{P} \mathcal{N}_{P} / T$, where $T$ is the time that the pump is on, $\mathcal{N}_{D} / T=2 P_{P} L^{2} /(\mathcal{P} \mathcal{A T})$ can be thought of as the average rate of generated photons provided that $|\beta| \sqrt{p_{\lambda}} \ll 1$, where $p_{\lambda}$ is the largest Schmidt coefficient in a Schmidt decomposition [12] of the biphoton wave function. For if one writes Eq. (5) in the basis of the Schmidt modes, and calculates the expectation value of the number of photons in Eq. (4), one sees that Eq. (10) remains true in the continuous wave $(\mathrm{CW})$ limit, provided $P_{P} \ll \mathcal{P} \mathcal{A} / L^{2}$. Defining $P_{I}=$ $\hbar \omega_{F} \mathcal{N}_{D} /(2 T)$ as the average generated power associated with one photon of each pair, we rewrite Eq. (10) as

$$
P_{I}=\frac{\hbar \omega_{F}}{\mathcal{T}} \frac{P_{P} L^{2}}{\mathcal{P} \mathcal{A}}
$$

To derive expressions for classical second-order nonlinear processes, we begin with the same Hamiltonian as above [Eq. (1)], Taylor expand the $\omega_{m k}$ to first order in the linear regime and zeroth order in the nonlinear regime, and introduce effective fields that are Schrödinger operators with an

$$
H_{2}^{N L}=-\frac{\bar{\chi}_{2}}{\bar{n}^{3} \sqrt{2 \varepsilon_{0} v_{\mathrm{SH}} v_{F}^{2} \mathcal{A}}} \int \mathrm{d} z G_{P}^{\dagger} G_{S} G_{I} e^{i\left(k_{S}+k_{I}-k_{P}\right) z}+\text { H.c., }
$$

where, so that we may compare a quantum (spontaneous) and a classical (stimulated) process each pumped at the same frequency, we have taken $\omega_{P}=\omega_{\mathrm{SH}}, v_{P}=v_{\mathrm{SH}}$, and $d_{P}^{i}(x, y)=$ $d_{\mathrm{SH}}^{i}(x, y)$, and assumed that the signal and generated idler frequencies are close enough to half the pump frequency that, outside the integral over $z, \omega_{S} \approx \omega_{I} \approx \omega_{P} / 2$ as well as $v_{S} \approx v_{I} \approx$ $v_{F}$ and $d_{S}^{i}(x, y) \approx d_{I}^{i}(x, y) \approx d_{F}^{i}(x, y)$. Coupled mode equations then follow from the Heisenberg equations of motion

$$
\begin{gathered}
\frac{1}{v_{\mathrm{SH}}} \frac{\partial G_{P}}{\partial t}+\frac{\partial G_{P}}{\partial z}=\frac{2 i}{\sqrt{\mathcal{P} \mathcal{A}}} G_{S} G_{I} e^{i\left(k_{S}+k_{I}-k_{P}\right) z}, \\
\frac{1}{v_{F}} \frac{\partial G_{S}}{\partial t}+\frac{\partial G_{S}}{\partial z}=\frac{i}{\sqrt{\mathcal{P A}}} G_{I}^{\dagger} G_{P} e^{-i\left(k_{S}+k_{I}-k_{P}\right) z}, \\
\frac{1}{v_{F}} \frac{\partial G_{I}}{\partial t}+\frac{\partial G_{I}}{\partial z}=\frac{i}{\sqrt{\mathcal{P A}}} G_{S}^{\dagger} G_{P} e^{-i\left(k_{S}+k_{I}-k_{P}\right) z},
\end{gathered}
$$


and, assuming a nonlinear region of length $L$, a CW DFG calculation in the undepleted pump (and signal) approximation for a channel waveguide yields

$$
P_{I}=P_{S} \frac{P_{P} L^{2}}{\mathcal{P} \mathcal{A}} \operatorname{sinc}^{2}\left\{\left[k_{S}\left(\omega_{S}\right)+k_{I}\left(\omega_{P}-\omega_{S}\right)-k_{P}\left(\omega_{P}\right)\right] \frac{L}{2}\right\}
$$

If we had instead followed the same steps and assumptions for a CW SHG calculation, we would have found

$$
P_{\mathrm{SH}}=\frac{P_{F}^{2} L^{2}}{\mathcal{P} \mathcal{A}} \operatorname{sinc}^{2}\left\{\left[k_{\mathrm{SH}}\left(2 \omega_{F}\right)-2 k_{F}\left(\omega_{F}\right)\right] \frac{L}{2}\right\},
$$

which agrees with the well-known result [13]: exact agreement is achieved if we set our $\mathcal{A}$ equal to the effective area presented there, and then, for we are free to do so, set $\bar{n}=$ $n$ and $\bar{\chi}_{2}=2 d_{i j k}^{\prime} / \varepsilon_{0}$, as well as approximate $v_{m} \approx c / n$.

Comparing our quantum [Eq. (12)] and classical [Eqs. (19) and (20)] expressions, we first note that SPDC, DFG, and SHG all scale the same way with the characteristic power, $\mathcal{P}$, and effective area, $\mathcal{A}$, of the device. But an important difference is the way in which energy conservation enters in these expressions. For CW pumps in classical (stimulated) experiments, the generated light appears at a single frequency that is tunable by varying the pump (and signal, in DFG) frequency, as determined by energy conservation. The efficiency of the process is determined by how well phase matching is achieved, in DFG for the so-determined idler frequency $\omega_{I}=\omega_{P}-\omega_{S}$, as expressed in the $\operatorname{sinc}^{2}$ function appearing in Eq. (19). On the contrary, in quantum (spontaneous) experiments, since only the energy sum of the signal and idler photons is fixed in the approximately CW experiments described by Eq. (12), there is a probability of photons being generated over a wide frequency range, centered about $\omega_{P} / 2$. This "spectral linewidth of emission" [14] is determined by $\mathcal{T}^{-1}$. Comparing Eq. (12) with Eq. (19) in the limit of perfect phase matching, we see that in the quantum case, the role of the classical "seed" power $P_{S}$ is played by $\hbar \omega_{F} / \mathcal{T}$, the average energy of one downconverted photon in a time $\mathcal{T}$, and that the wider the bandwidth of possible emission, the larger the fluctuating power available to drive the process.

Turning now to an evaluation of $\mathcal{T}$, if the phase-matching condition is satisfied over a range that is small compared to the range of integration, i.e., when $1-\left(2 \Omega / \omega_{P}\right)^{2}$ remains essentially constant over the frequency range within which the squared sinc function is significantly nonzero, we have

$$
\mathcal{T} \approx \frac{2 \pi}{\int_{0}^{\omega_{P} / 2} \mathrm{~d} \Omega \operatorname{sinc}^{2}\left\{\left[k_{F}\left(\omega_{P} / 2-\Omega\right)+k_{F}\left(\omega_{P} / 2+\Omega\right)-k_{\mathrm{SH}}\left(\omega_{P}\right)\right] L / 2\right\}} .
$$

Taylor expanding dispersion relations to quadratic order about the phase-matched case, i.e.,

$$
k_{m}(\omega)=k_{m}\left(\omega_{m_{0}}\right)+\frac{1}{v_{m}}\left(\omega-\omega_{m_{0}}\right)+\frac{\beta_{2}\left(\omega_{m_{0}}\right)}{2}\left(\omega-\omega_{m_{0}}\right)^{2},
$$

we find

$$
\mathcal{T} \approx \frac{3}{2} \sqrt{2 \pi\left|\beta_{2}\left(\omega_{P} / 2\right)\right| L}
$$

from Eq. (21) for a pump centered at the second harmonic, $\omega_{P}=\omega_{\mathrm{SH}_{0}}$, and thus, inserting Eq. (르) into Eq. (느), we obtain

$$
P_{I}=\frac{\hbar \omega_{F}}{\frac{3}{2} \sqrt{2 \pi\left|\beta_{2}\left(\omega_{P} / 2\right)\right| L}} \frac{P_{P} L^{2}}{\mathcal{P} \mathcal{A}} .
$$

Since the time $\mathcal{T}$ is determined by material dispersion, it is proportional to $L^{1 / 2}$. Therefore the generated power scales as $L^{3 / 2}$ in an SPDC experiment, and not with the square of the device length as it does in DFG. We note that the spontaneous expression (12), with $\mathcal{T}$ given by Eq. (21), agrees with a previously published result [14]: exact agreement is achieved if we set our $\mathcal{A}$ equal to the effective area presented there, and then, for we are free to do so, set $\bar{n}=\left(n_{S} n_{I} n_{P}\right)^{1 / 3}$ and $\bar{\chi}_{2}=2 d_{\text {eff }} / \varepsilon_{0}$, and approximate $v_{m} \approx c / n_{m}$, as well as $\omega_{I} \approx$ $\omega_{S}=\omega_{F}$, similar to above.

So far we have assumed that the SPDC is unfiltered. If a bandpass filter with bandwidth $\Delta \omega=2 \pi B$ is used, narrow enough that the sinc function varies negligibly over that range, the time $\mathcal{T}$ of Eq. (21) becomes

$\mathcal{T}(\Omega) \approx\left(B \operatorname{sinc}^{2}\left\{\left[k_{F}\left(\omega_{P} / 2-\Omega\right)+k_{F}\left(\omega_{P} / 2+\Omega\right)-k_{\mathrm{SH}}\left(\omega_{P}\right)\right] L / 2\right\}\right)^{-1}$,

where $\Omega+\omega_{P} / 2$ identifies the center frequency of the bandpass filter, and the generated power

$$
\begin{aligned}
P_{I}(\Omega)= & \frac{\hbar \omega_{F}}{B^{-1}} \frac{P_{P} L^{2}}{\mathcal{P} \mathcal{A}} \operatorname{sinc}^{2}\left\{\left[k_{F}\left(\omega_{P} / 2-\Omega\right)\right.\right. \\
& \left.\left.+k_{F}\left(\omega_{P} / 2+\Omega\right)-k_{\mathrm{SH}}\left(\omega_{P}\right)\right] \frac{L}{2}\right\}
\end{aligned}
$$

scales with $L^{2}$ as in the classical expression. Note that when comparing DFG [Eq. (19)] to this expression [Eq. (26)], it is not just the efficiency of the process that has changed-reduced by a factor of $\hbar \omega_{F} /\left(B^{-1} P_{S}\right)$-but also the frequency of generated light has gone from being determined by $\omega_{I}=$ $\omega_{P}-\omega_{S}$ to being a spectral density. And so here it is by tuning $\Omega$ that the idler frequency is selected, rather than by tuning $\omega_{S}$ as in the stimulated (DFG) scenario.

Finally, it is interesting to consider the artificial scenario in which there is no dispersion and the interacting fields are perfectly phase matched. In practice, this assumption may not be so unrealistic, for if the system length $L$ is much shorter than the coherence length $L_{C}=\pi /\left[k_{F}\left(\omega_{P} / 2-\Omega\right)+k_{F}\left(\omega_{P} / 2+\Omega\right)-\right.$ $k_{\mathrm{SH}}\left(\omega_{P}\right)$ ] over the integration range of Eq. (11), where the modes exist, then the sinc function is essentially unity everywhere there. Of course, an actual calculation would have to take into account mode cut-offs in limiting the range of integration [9]. But if we further neglect those cut-offs and assume that the modes exist over the entire integration range of Eq. (11), then if there is no filtering we find that the generation bandwidth time is on the order of the period of a generated photon $\mathcal{T}=3 \pi /\left(\omega_{P} / 2\right)$, so that

$$
P_{I}=\frac{\hbar \omega_{F}}{\frac{3}{2} \tau_{F}} \frac{P_{P} L^{2}}{\mathcal{P} \mathcal{A}},
$$

where we have identified $\tau_{m}=2 \pi / \omega_{m}$ as a typical photon period. Unlike in Eq. (24), here the output also scales as $L^{2}$, for 
there is no length dependent bandwidth over which the output is generated.

With these considerations, determination of the material parameters $L^{2} /(\mathcal{P} \mathcal{A})$ in a classical experiment (here, DFG or SHG) allows for an accurate prediction of the average power of photons generated via SPDC.

\section{B. Third-Order Processes}

The method and results of this section closely parallel that of the second-order case. Because they have not yet been reported, we include the mathematical details in Appendices $\underline{\mathrm{A}}$ and $\underline{\mathrm{B}}$. We begin with the Hamiltonian

$$
\begin{gathered}
H_{3}=H_{L}+H_{N L}, \\
H_{L}=\int \mathrm{d} k \hbar \omega_{k} a_{k}^{\dagger} a_{k}, \\
H_{N L}=-\int \mathrm{d} k_{1} \mathrm{~d} k_{2} \mathrm{~d} k_{3} \mathrm{~d} k_{4} S\left(k_{1}, k_{2}, k_{3}, k_{4}\right) a_{k_{1}}^{\dagger} a_{k_{2}}^{\dagger} a_{k_{3}} a_{k_{4}}+\text { H.c., }
\end{gathered}
$$

where $S\left(k_{1}, k_{2}, k_{3}, k_{4}\right)$ is a nonlinear coupling term depending on an effective area as well as a phase-matching function [9], as in Eq. (3) above [see Eqs. (1ㅡ) and (2) in Appendix $\underline{\mathrm{A}}$ ].

Following the backward Heisenberg picture approach [ㅁ] and the same arguments as above about the low-power limit in which the result is valid, we calculate the average power associated with one photon of each pair generated due to a CW SFWM process in a channel waveguide pumped at $\omega_{P}$ to be

$$
P_{I}=\frac{\hbar \omega_{P}}{\mathcal{T}}\left(\gamma P_{P} L\right)^{2}
$$

where $L$ is the length of the nonlinear structure, and $\gamma$ is the usual nonlinear parameter [15], written in our notation in Appendix $\underline{\mathrm{A}}, P_{P}=\hbar \omega_{P} \mathcal{N}_{P} / T$ and $P_{I}=\hbar \omega_{P} \mathcal{N}_{D} /(2 T)$, analogous to the expressions preceding Eq. (12), with the generation bandwidth time

$\mathcal{T}=\frac{2 \pi}{\int_{0}^{\omega_{P}} \mathrm{~d} \Omega\left[1-\left(\Omega / \omega_{P}\right)^{2}\right] \operatorname{sinc}^{2}\left\{\left[2 k\left(\omega_{P}\right)-k\left(\omega_{P}+\Omega\right)-k\left(\omega_{P}-\Omega\right)\right] L / 2\right\}}$,

and $\Omega=\omega_{1}-\omega_{P}$ representing positive detuning from $\omega_{P}$. This result is in a form that anticipates the classical FWM result, namely

$P_{I}=P_{S}\left(\gamma P_{P} L\right)^{2} \operatorname{sinc}^{2}\left\{\left[2 k_{P}\left(\omega_{P}\right)-k_{S}\left(\omega_{S}\right)-k_{I}\left(2 \omega_{P}-\omega_{S}\right)\right] L / 2\right\}$.

The classical equation is derived from the same Hamiltonian as the quantum equation (28), and for the mathematical details we refer the reader to Appendix B.

Comparing the corresponding results here, we see that there is a clear analogy with the comparison of second-order processes in that the classical (stimulated) process leads to the generation of a fixed idler frequency, for set pump and signal frequencies, with an efficiency determined by the familiar phase-matching function. Similarly, in the quantum (spontaneous) process, the signal and idler photons can be generated over a range of frequencies determined by a bandwidth $\mathcal{T}^{-1}$. Comparing Eq. (33) in the limit of perfect phase matching with Eq. (31), we see that the role of the seed power $P_{S}$ in the classical (stimulated) process is played by $\hbar \omega_{P} / \mathcal{T}$ in the quantum (spontaneous) process, the average energy of one generated photon in a time $\mathcal{T}$, and that the wider the bandwidth of possible emission, the larger the fluctuating power available to drive the process.

We now turn to an evaluation of $\mathcal{T}$ [Eq. (32)]. If the phasematching condition is satisfied over a range that is small compared to the range of integration, we note that $1-\left(\Omega / \omega_{P}\right)^{2}$ stays very close to 1 over the range of frequencies in which the squared sinc function is significantly nonzero, and Taylor expansion of the dispersion relation about $\omega_{P}$,

$$
k(\omega)=k\left(\omega_{P}\right)+\frac{1}{v}\left(\omega-\omega_{P}\right)+\frac{\beta_{2}\left(\omega_{P}\right)}{2}\left(\omega-\omega_{P}\right)^{2},
$$

in Eq. (32) leads to

$$
\mathcal{T} \approx \frac{3}{2} \sqrt{2 \pi\left|\beta_{2}\left(\omega_{P}\right)\right| L}
$$

This is essentially the same expression found above for the SPDC generation bandwidth time (23) when generated photons are collected over the entire available bandwidth. Substituting Eq. (35) in Eq. (31), we obtain

$$
P_{I}=\frac{\hbar \omega_{P}}{\frac{3}{2} \sqrt{2 \pi\left|\beta_{2}\left(\omega_{P}\right)\right| L}}\left(\gamma P_{P} L\right)^{2} .
$$

Since the time $\mathcal{T}$ is determined by material dispersion, it is proportional to $L^{1 / 2}$, and therefore the generated power scales as $L^{3 / 2}$ in an SFWM experiment, and not with the square of the device length as it does in FWM. We note that Eqs. (31), (35), and (33) agree with well-known results [15,16].

If instead of looking at the entire SFWM spectrum, we choose to focus on a narrow spectral region of bandwidth $2 \pi B$; we find

$$
\mathcal{T}(\Omega) \approx\left(B \operatorname{sinc}^{2}\left\{\left[2 k\left(\omega_{P}\right)-k\left(\omega_{P}+\Omega\right)-k\left(\omega_{P}-\Omega\right)\right] L / 2\right\}\right)^{-1} .
$$

Under this assumption, for a particular detuning $0 \leq \Omega \leq \omega_{P}$, from $\omega_{P}$, the generated power can be written

$P_{I}(\Omega)=\frac{\hbar \omega_{P}}{B^{-1}}\left(\gamma P_{P} L\right)^{2} \operatorname{sinc}^{2}\left\{\left[2 k\left(\omega_{P}\right)-k\left(\omega_{P}+\Omega\right)-k\left(\omega_{P}-\Omega\right)\right] \frac{L}{2}\right\}$,

and scales with $L^{2}$ as in the classical expression.

In the somewhat artificial scenario in which the system length is much smaller than the coherence length $L_{C}=$ $\pi /\left[2 k\left(\omega_{P}\right)-k\left(\omega_{P}+\Omega\right)-k\left(\omega_{P}-\Omega\right)\right]$ over the relevant range of integration (32), where again we neglect the fact that in a real calculation some of this integration range will not contribute due to mode cut-offs, we find $\mathcal{T}=3 \pi / \omega_{P}$ if there is no filtering, and the generated power becomes 
Table 1. Expressions for the Power Generated in Corresponding Nonlinear Optical Processes in Channel Waveguides

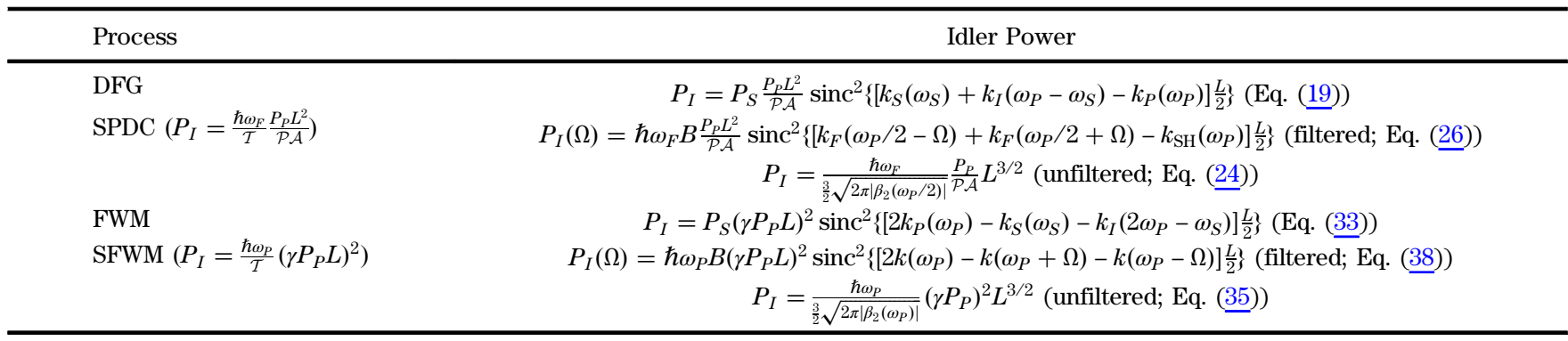

$$
P_{I}=\frac{\hbar \omega_{P}}{\frac{3}{2} \tau_{P}}\left(\gamma P_{P} L\right)^{2},
$$

where $\tau_{P}=2 \pi / \omega_{P}$ is a typical photon period. Unlike in Eq. (36), here the output also scales as $L^{2}$, for there is no length dependent bandwidth over which the output is generated.

We conclude this section by summarizing all of the results for spontaneous and stimulated processes in a channel waveguide. If one wishes to make a quick estimate of the average generated photon power at a certain input power, in either an SPDC or SFWM experiment, one can simply perform the corresponding classical experiment, extract the relevant material and structure parameters, and lastly replace $P_{S}$ with the energy of a typical generated photon $\left(\hbar \omega_{P} / 2\right.$ or $\hbar \omega_{P}$, for SPDC or SFWM, respectively) divided by the generation bandwidth time $\mathcal{T}$ and adjusting accordingly for any filtering before detection. If the power can be collected over the entire generation bandwidth, then Eqs. (24) or (35) should be used. It is interesting to note that for the spontaneous processes in the short system length limit $L \ll L_{C}$, if mode cut-offs are neglected, a power of $2 \hbar \omega /(3 \tau)$ (about $26 \mu \mathrm{W}$ for a $1 \mathrm{eV}$ photon) plays the role of the classical "seed" power of the corresponding classical calculation for both SPDC [Eq. (27)] and SFWM [Eq. (39)].

The expressions required for such estimates (the "idler" powers generated in corresponding second- and third-order nonlinear processes) are shown in Table 1 . In this table, we have chosen to rewrite the expressions to highlight the dependence on quantities that might be of interest in guiding the design of a device or in helping the interpretation of experimental results. Interestingly, for a channel waveguide, the scaling behavior of either stimulated or spontaneous processes with the length $L$ of the channel is independent of the order of the nonlinearity. In particular, the efficiency of phase-matched stimulated generation scales with $L^{2}$ whether we are considering DFG or FWM. The same scaling is obtained for the generation rate of spontaneous processes within a sufficiently narrow spectral region around the phase-matching condition. Similarly, the generated idler power, integrated over the entire spectrum, scales with $L^{3 / 2}$ for both SPDC and SFWM, as in the absence of filtering the generation bandwidth time scales as $L^{1 / 2}$. Of the quantum expressions, we note that the bandpass filtered expression is most often investigated experimentally, especially for third-order nonlinear devices where strong pump suppression is required and Raman noise should be avoided
[17], and so $L^{2}$ scaling is typically observed. Finally, considering the field enhancement due to the light confinement in the channel, which is inversely proportional to the effective area, we observe that the generated power is independent of the nature of the process, stimulated or spontaneous, while it depends upon the order of the nonlinearity. Indeed, for DFG and SPDC, the generation rate is proportional to $1 / \mathcal{A}$, while for FWM and SFWM it scales with $\gamma^{2} \propto 1 / \mathcal{A}^{2}$.

\section{RESONANT CASE}

In this section, we consider corresponding processes in a resonant structure, in particular a microring resonator sidecoupled to a channel waveguide [18-20]. Nonlinear processes can be largely amplified near particular frequencies when large field enhancements are achieved due to constructive interference of the electromagnetic field inside the ring. Thus, compared to processes in channel waveguides, we expect to be able to generate the same idler power with reduced pump (and signal) power(s) at particular frequencies. Furthermore, in a high- $Q$ ring resonator, we expect the generation bandwidth time to reflect the fact that this enhancement occurs over a series of narrow frequency ranges associated with the ring resonances, separated by the free spectral range (FSR) of the ring. As above, we first consider SPDC, DFG, and SHG before moving on to $\chi_{3}$ processes.

Table 2. Expressions for the Power Generated in Corresponding Nonlinear Optical Processes in Microring Resonators

\begin{tabular}{|c|c|}
\hline Process & Idler Power \\
\hline DFG & $\begin{array}{c}P_{I}=P_{S} \frac{P_{P}}{\mathcal{P}} \frac{32 v_{\mu S} v_{\mu I} v_{\mu P}}{\pi \omega_{\mu S} \omega_{\mu I} \omega_{\mu P} \mathcal{A}}\left(\frac{Q_{\mu S} Q_{\mu I} Q_{\mu P}}{R}\right) \\
\text { (Eq. (51)) }\end{array}$ \\
\hline $\operatorname{SPDC}\left(P_{I}=\frac{\hbar \omega_{\mu_{F}}}{\mathcal{T}} \frac{P_{P}}{\mathcal{P}} \frac{8 \pi v_{\mu P} P}{\omega_{\mu P \mathcal{A}}} R Q_{\mu P}\right)$ & $\begin{array}{c}P_{I}(\Omega)=\hbar B \frac{P_{P}}{\mathcal{P}} \frac{32 v_{\mu} v_{\mu F}^{2}}{\pi \omega_{\mu P} \omega_{\mu F} \mathcal{A}}\left(\frac{Q_{\mu P} Q_{\mu F}^{2}}{R}\right) \\
\text { (filtered; Eq. }(\underline{56)})) \\
P_{I}=\hbar \frac{P_{P}}{\mathcal{P}} \frac{4 v_{\mu p} v_{\mu F}^{2}}{\pi \omega_{\mu P} \mathcal{A}}\left(\frac{Q_{\mu F} Q_{\mu P}}{R}\right) \\
\text { (unfiltered; Eq. }(\underline{55}))\end{array}$ \\
\hline FWM & $\begin{array}{c}P_{I}=P_{S}\left(\gamma P_{P}\right)^{2} \frac{64 v_{\mu \nu}^{2} v_{\mu S} v_{\mu I}}{\pi^{2} \omega_{\mu P}^{2} \omega_{\mu S} \omega_{\mu I}}\left(\frac{Q_{\mu P}^{2} Q_{\mu S} Q_{\mu I}}{R^{2}}\right) \\
\text { (Eq. }(\underline{60)}))\end{array}$ \\
\hline $\begin{array}{l}\text { SFWM }\left(P_{I}=\overline{\bar{h}}_{\frac{\hbar \omega_{\mu P}}{T}}^{\mathcal{T}}\left(\gamma P_{P}\right)^{2} \frac{16 v_{\mu P}}{\omega_{\mu P}^{2}} Q_{\mu P}^{2}\right)\end{array}$ & $\begin{array}{c}P_{I}(\Omega)=\hbar B\left(\gamma P_{P}\right)^{2} \frac{64 v_{\mu P}^{4}}{\pi^{2} \omega_{\mu P}^{3}}\left(\frac{Q_{\mu P}^{4}}{R^{2}}\right) \\
\text { (filtered; Eq. } \underline{(63)}) \\
P_{I}=\hbar\left(\gamma P_{P}\right)^{2} \frac{8 v_{\mu P}^{4}}{\pi^{2} \omega_{\mu P}^{2}}\left(\frac{Q_{\mu P}^{3}}{R^{2}}\right) \\
\text { (unfiltered; Eq. }(\underline{62}))\end{array}$ \\
\hline
\end{tabular}




\section{A. Second-Order Processes}

We treat optical dynamics in a microring resonator sidecoupled to a channel waveguide when only the second-order optical nonlinearity is relevant with the Hamiltonian [21]

$$
\begin{gathered}
H=H_{L}+H_{N L}, \\
H_{L}=H_{\mathrm{ch}}+H_{r}+H_{\mathrm{cp}}, \\
H_{L}=-\sum_{\mu 1 . \mu 2}\left[S_{\mu_{1} \mu_{2} \mu_{\mathrm{SH}}} b_{\mu_{\mathrm{SH}}^{\dagger}} b_{\mu_{1}} b_{2}+\text { H.c. }\right], \\
H^{\mathrm{ch}}=\sum_{\mu}\left[\hbar \omega_{\mu} \int \mathrm{d} z \psi_{\mu}^{\dagger}(z) \psi_{\mu}(z)\right. \\
\left.+\frac{i}{2} \hbar v_{\mu} \int \mathrm{d} z\left(\frac{\partial \psi_{\mu}^{\dagger}(z)}{\partial z} \psi_{\mu}(z)-\text { H.c. }\right)\right], \\
H^{\mathrm{cp}}=\sqrt{2 \pi} \hbar \sum_{\mu}\left[c_{\mu} b_{\mu} \psi_{\mu}^{\dagger}(0)+\text { H.c. }\right], \\
H^{R}=\sum_{\mu} \hbar \omega_{\mu} b_{\mu}^{\dagger} b_{\mu},
\end{gathered}
$$

where, similar to above, all of the nonlinearity has been lumped into $S_{\mu_{1} \mu_{2} \mathrm{SH}} ; c_{\mu}$ is a coupling constant defined later, the $b_{\mu}$ are ring-mode operators, and the $\psi_{\mu}$ are effective field operators for the channel, though without any explicit time dependence or scaling to create units of power as for the $G_{m}$ above. In the channel we have split the $k$ spectrum into discrete regions, each in the neighborhood of a ring resonance. Provided that the range of $k$ covered by the pump (and seed) launched into the channel is much narrower than the range of $k$ used to define a single resonance region (i.e., that the ring has a large FSR compared to the pump bandwidth), we may treat the coupling between the channel and the ring as connecting a single $\mu$ from the channel to the corresponding $\mu$ within the ring. Thus, we use $\mu$ to label the modes instead of the bare $m$ above to indicate ring resonance order $N$, i.e., $\mu=(m, N)$, with a resonance defined according to

$$
k_{\mu} \cdot R=N \text {, }
$$

where $R$ is the ring radius.

While our formalism can treat nonlinear susceptibility tensors of general symmetry, for instance, rings made of III-V semiconductors in which there are unusual quasiphase-matching conditions [11], for the sake of simplicity, we here assume that the nonlinearity experienced by the field of a ring mode remains constant as that field circles the ring. We also assume that the fields involved are at frequencies near resonances, leading to strong field enhancement due to constructive interference in the ring resonator and allowing us to safely neglect any nonlinear effects outside the ring in the waveguide, where such enhancement does not take place. To quantify how close the fields are to ring resonances, consider first the example of SHG where we identify the $k_{\mu}$ [Eq. (ㄷ)] associated with the fundamental field as $k_{\mu_{F}}$, and that with the second harmonic field as $k_{\mu_{\mathrm{SH}}}$; we assume these are chosen such that $\left(2 k_{\mu_{F}}-k_{\mu_{\mathrm{SH}}}\right) R=2 N_{F}-N_{\mathrm{SH}}=0$, where $N_{F}$ and $N_{\mathrm{SH}}$ are the integers associated [Eq. (46)] with $k_{\mu_{F}}$ and $k_{\mu_{\mathrm{SH}}}$, respectively. For the frequencies, $\omega_{\mu_{F}}$ and $\omega_{\mu_{\mathrm{SH}}}$ associated with these modes [Eq. (45)], in general we will have $\omega_{\mu_{\mathrm{SH}}} \neq$ $2 \omega_{\mu_{F}}$ because of dispersion, but we will assume that the fundamental and second harmonic frequencies $\omega_{F}$ and $\omega_{\mathrm{SH}}$, respectively, with $\omega_{\mathrm{SH}}=2 \omega_{F}$, are characterized by detunings $\delta_{\mu_{F(\mathrm{SH})}}=\left|\omega_{F(\mathrm{SH})}-\omega_{\mu_{F(\mathrm{SH})}}\right|$ so small that $2 \pi R \delta_{\mu_{F(\mathrm{SH})}} / v_{\mu_{F(\mathrm{SH})}} \ll 1$.

To make a CW SPDC calculation, we follow the approach outlined in [21] in the limit of a long pump pulse. As discussed above, this is easily generalized to a CW pump provided that the pump power is kept low enough. We consider a pump at $\omega_{P}$, chosen to be the center frequency $\omega_{\mu_{\mathrm{SH}}}$ of the ring mode identified by the waveunumber $k_{\mu_{\mathrm{SH}}}$ defined above, and write $\omega_{P}$ as $\omega_{P} \equiv \omega_{\mu_{\mathrm{SH}}}$. For photons generated within a pair of resonances near enough to $N_{F}=N_{P} / 2$, one less and one greater than $N_{P} / 2$, where $N_{P} \equiv N_{\mathrm{SH}}$, that we may take $v_{\mu_{1}} \approx v_{\mu_{2}} \approx v_{\mu_{F}}$ and $d_{\mu_{1}}^{i}(x, y) \approx d_{\mu_{2}}^{i}(x, y) \approx d_{\mu_{F}}^{i}(x, y)$, as well as $\omega_{\mu_{1}} \approx \omega_{\mu_{2}} \approx \omega_{\mu_{F}}$, we find that the average generated power associated with one photon of each pair is

$$
P_{I}=\frac{\hbar \omega_{\mu_{F}}}{\mathcal{T}} \frac{P_{P} L^{2}}{\mathcal{P} \mathcal{A}}\left|F_{\mu_{P}}\left(\omega_{\mu_{P}}\right)\right|^{2}
$$

Here $L=2 \pi R, \mathcal{P}$, and $\mathcal{A}$ are as above [Eq. (12)], though now defined in terms of a specific set of center frequencies, and the

$$
F_{\mu}(\omega)=\frac{i\left[2\left(1-\sigma_{\mu}\right)\right]^{1 / 2}}{\left(1-\sigma_{\mu}\right)-i\left(\omega-\omega_{\mu}\right) L / v_{\mu}},
$$

are field enhancement factors with $\sigma_{\mu}$ the usual transmission or "self-coupling" constant related to the coupling constant in the Hamiltonian above via $\sigma_{\mu}=1-\pi\left|c_{\mu}\right|^{2} L / v_{\mu}^{2}$ [21] . In particular, when the frequency is at a ring resonance frequency, $\omega=\omega_{\mu}$, we can write the enhancement factors in terms of the quality factor(s) of the ring

$$
F_{\mu}\left(\omega_{\mu}\right)=2 i \sqrt{\frac{Q_{\mu} v_{\mu}}{\omega_{\mu} L}} .
$$

In the limit of no loss, small detuning, and weak coupling $\left(\sigma_{\mu} \approx 1\right)$, these field enhancement factors are equivalent to those defined in [22]. Recalling that we take the generated photon resonances close to $N_{P} / 2$, we can take $F_{\mu_{1}}(\omega) \approx F_{\mu_{2}}(\omega) \approx F_{\mu_{F}}(\omega)$, and find that the generation bandwidth time across a single resonance is

$$
\mathcal{T}=\frac{2 \pi}{\int \mathrm{d} \omega\left|F_{\mu_{F}}\left(\omega_{\mu_{P}} / 2-\omega\right)\right|^{2}\left|F_{\mu_{F}}\left(\omega_{\mu_{P}} / 2+\omega\right)\right|^{2}} .
$$

Following a similar approach to the channel calculation above, a CW DFG calculation yields

$$
P_{I}=P_{S} \frac{P_{P} L^{2}}{\mathcal{P} \mathcal{A}}\left|F_{\mu_{S}}\left(\omega_{S}\right)\right|^{2}\left|F_{\mu_{I}}\left(\omega_{P}-\omega_{S}\right)\right|^{2}\left|F_{\mu_{P}}\left(\omega_{P}\right)\right|^{2},
$$

whereas the SHG result is 


$$
P_{\mathrm{SH}}=\frac{P_{F}^{2} L^{2}}{\mathcal{P} \mathcal{A}}\left|F_{\mu_{F}}\left(\omega_{F}\right)\right|^{4}\left|F_{\mu_{\mathrm{SH}}}\left(2 \omega_{F}\right)\right|^{2} .
$$

We note that [Eq. (52)] was presented earlier [11], with consideration for arbitrary detuning from resonance, for a GaAs ring resonator in which quasi-phase matching must be taken into account. We further note that our strategy for labeling the modes and assumption that all fields are nearly on resonance has eliminated from these results a squared sinc function that might have been expected. Were we to make this calculation without a Hamiltonian formalism that uses ring resonances as a set of modes and eliminates the sinc function as in, for instance, [11], this assumption would mean that the squared sinc function would essentially evaluate to 1 .

We now turn to a comparison of the classical (stimulated) processes described by Eqs. (51) and (52), and the quantum (spontaneous) process described by Eq. (그). Note first that, setting aside intensity enhancement factors, there is a quantum power $\hbar \omega_{\mu_{F}} / \mathcal{T}$ that plays the role of the seed power $P_{S}$ in Eq. (51), analogous to what was seen in comparing SPDC [Eq. (12)] with DFG [Eq. (19)] in a channel waveguide. For the structures considered here, there is an intensity enhancement factor for the pump $\left|F_{\mu_{P}}\left(\omega_{P}\right)\right|^{2}$ [two, resulting in $\left|F_{\mu_{F}}\left(\omega_{F}\right)\right|^{4}$ in Eq. (52)] appearing in all three expressions (47), (51), and (52). In the stimulated processes, we also have intensity enhancement factors associated with the signal input and idler output in Eq. (51), and with the second harmonic output in Eq. (52). In Eq. (47), which describes an unseeded process, the frequency enhancement factors associated with the generated fields reside in the generation bandwidth time $\mathcal{T}$, and reflect the fact that there is a range of frequencies over which the generated photons can appear. Unlike in the channel calculation, that time can be calculated here exactly, under the mild assumption that the integration range over the Lorentzians can be extended to infinity without serious error. The result is

$$
\mathcal{T}=\frac{\left|F_{\mu_{F}}\left(\omega_{\mu_{F}}\right)\right|^{4} L^{3}\left(2 \omega_{\mu_{F}}-\omega_{\mu_{F}}\right)^{2}+16 v_{\mu_{F}}^{2} L}{8 v_{\mu_{F}}^{3}\left|F_{\mu_{F}}\left(\omega_{\mu_{F}}\right)\right|^{2}},
$$

where recall $\omega_{\mu_{P}}$ is the center frequency of the ring resonance at which the pump frequency $\omega_{P}$ is also centered, and $\omega_{\mu_{F}}$ is the center frequency of the ring resonance in which $\omega_{P} / 2$ resides. We see that generation efficiency is worse the farther $\omega_{\mu_{F}}$ is from $\omega_{\mu_{P}} / 2$. For $\omega_{\mu_{F}}$ sufficiently close to $\omega_{\mu_{P}} / 2$, we have

$$
\mathcal{T}=\frac{2 L}{v_{\mu_{F}}\left|F_{\mu_{F}}\left(\omega_{\mu_{F}}\right)\right|^{2}}=\frac{\omega_{\mu_{F}} L^{2}}{2 v_{\mu_{F}}^{2} Q_{\mu_{F}}}
$$

where we have used Eq. (49). We note that a similar result, albeit in a slightly different form and calculated for a GaAs add/drop ring resonator instead of a ring resonator sidecoupled to a single channel waveguide, was previously presented [21]. Substituting Eq. (4) in Eq. (느), we find

$$
\begin{aligned}
P_{I} & =\frac{\hbar \omega_{\mu_{F}} v_{\mu_{F}}}{2 L\left|F_{\mu_{F}}\left(\omega_{\mu_{F}}\right)\right|^{2}} \frac{P_{P} L^{2}}{\mathcal{P} \mathcal{A}}\left|F_{\mu_{P}}\left(\omega_{\mu_{P}}\right)\right|^{2}\left|F_{\mu_{F}}\left(\omega_{\mu_{F}}\right)\right|^{4} \\
& =\frac{\hbar \omega_{\mu_{F}}}{\frac{Q_{\mu_{F}}}{16 \pi} \tau_{\mu_{F}}} \frac{P_{P} L^{2}}{\mathcal{P} \mathcal{A}} \frac{Q_{\mu_{P}} v_{\mu_{P}}}{\omega_{\mu_{P}} L}\left(\frac{Q_{\mu_{F}} v_{\mu_{F}}}{\omega_{\mu_{F}} L}\right)^{2},
\end{aligned}
$$

where we have introduced $\tau_{\mu_{F}}=2 \pi / \omega_{\mu_{F}}$. On the basis of Eqs. (51) and (52), and the fact the intensity enhancement factors $\left|\overline{F_{\mu}}\left(\omega_{\mu}\right)\right|^{2}$ are proportional to the $Q_{\mu}(\underline{49})$, one might have expected to see three quality factors in Eq. (55). However, because of the resonance, the generation bandwidth time is also increased by a factor of $Q_{\mu_{F}}$ and so there are, in the end, only two that result.

Recall that we have assumed that all of the light is collected over a full ring resonance linewidth. If one had a narrow enough filter with bandwidth $2 \pi B$ that would select out photons generated within just a small window of a ring resonance, the associated calculation would yield

$$
\begin{aligned}
P_{I}(\Omega)= & \frac{\hbar \omega_{\mu_{F}}}{B^{-1}} \frac{P_{P} L^{2}}{\mathcal{P} \mathcal{A}}\left|F_{\mu_{P}}\left(\omega_{\mu_{P}}\right)\right|^{2}\left|F_{\mu_{F}}\left(\omega_{\mu_{P}} / 2-\Omega\right)\right|^{2} \\
& \left|F_{\mu_{F}}\left(\omega_{\mu_{P}} / 2+\Omega\right)\right|^{2},
\end{aligned}
$$

where $\Omega+\omega_{\mu_{P}} / 2$ now represents the center frequency of the accepted light.

It is worth noting that there is an entire frequency "comb" of resonances on either side of half of the resonance being pumped that essentially satisfies both energy and momentum conservation for the generated correlated photon pairs. Generation is significant up until $\omega_{\mu_{S}}+\omega_{\mu_{I}}-\omega_{\mu_{P}}$ becomes too large. Quantifying this a bit more, the generation rate will be reduced by a factor of 2 when $\omega_{\mu_{S}}+\omega_{\mu_{I}}-\omega_{\mu_{P}}=$ $4 v_{\mu_{F}} /\left(\left|F_{\mu_{F}}\left(\omega_{\mu_{F}}\right)\right|^{2} L\right)=\omega_{\mu_{F}} / Q_{\mu_{F}}$. Taylor expanding $\omega_{(m, N)}=$ $\omega_{\left(m, N_{m}\right)}+v_{m}\left(N-N_{m}\right) / R+\Xi_{m}\left(N-N_{m}\right)^{2} / R^{2}$, and remembering that if $\omega_{\mu_{S}}$ is associated with resonance order $N$ that $\omega_{\mu_{I}}$ is associated with resonance order $N_{P}-N$, we find $\omega_{\mu_{\mathrm{S}}}+$ $\omega_{\mu_{I}}-\omega_{\mu_{P}}=2 \Xi_{F}\left(N-N_{F}\right)^{2} / R^{2}$. Thus, when

$$
N-N_{F}=R\left[\omega_{\mu_{F}} /\left(2\left|\Xi_{F}\right| Q_{\mu_{F}}\right)\right]^{1 / 2},
$$

the generation rate drops by a factor of 2 .

Armed with Eqs. (51), (47), and (56), a classical (namely, DFG or SHG) experiment that determines $L^{2} /(\mathcal{P} \mathcal{A})$ as well as the strength of a typical enhancement factor, $\left|F_{\mu_{F}}\left(\omega_{\mu_{F}}\right)\right|^{2}$, allows for an accurate prediction of the average power of photons generated in a corresponding quantum experiment.

\section{B. Third-Order Processes}

The main results of this subsection were first presented in [23], and so we direct the reader there for details. Again we assume that the pump frequency $\omega_{P}$ is at the center of one of the ring resonances, $\omega_{P}=\omega_{\mu_{P}}$, and we find the average power associated with half of the generated photons to be

$$
P_{I}=\frac{\hbar \omega_{\mu_{P}}}{\mathcal{T}}\left(\gamma P_{P} L\right)^{2}\left|F_{\mu_{P}}\left(\omega_{\mu_{P}}\right)\right|^{4},
$$

where we have assumed that we are looking at photons generated within a pair of resonances near enough to $N_{P}$ that we may take $v_{\mu_{S}} \approx v_{\mu_{I}} \approx v_{\mu_{P}}$ and $d_{\mu_{S}}^{i}(x, y) \approx d_{\mu_{I}}^{i}(x, y) \approx d_{\mu_{P}}^{i}(x, y)$, as well as $\omega_{\mu_{S}} \approx \omega_{\mu_{I}} \approx \omega_{\mu_{P}}$, and $F_{\mu_{S}}(\omega) \approx F_{\mu_{I}}(\omega) \approx F_{\mu_{P}}(\omega)$, with a generation bandwidth time of

$$
\mathcal{T}=\frac{2 \pi}{\int \mathrm{d} \omega\left|F_{\mu_{P}}\left(\omega_{\mu_{P}}-\omega\right)\right|^{2}\left|F_{\mu_{P}}\left(\omega_{\mu_{P}}+\omega\right)\right|^{2}} .
$$

The corresponding CW FWM calculation yields 


$$
P_{I}=P_{S}\left(\gamma P_{P} L\right)^{2}\left|F_{\mu_{P}}\left(\omega_{P}\right)\right|^{4}\left|F_{\mu_{S}}\left(\omega_{S}\right)\right|^{2}\left|F_{\mu_{I}}\left(2 \omega_{P}-\omega_{S}\right)\right|^{2},
$$

where $\gamma$ is as above, only now defined in terms of a specific set of center frequencies. We note that this expression agrees with a well-known result [22], in the limit of no loss, small detuning, and weak coupling $\left(\sigma_{\mu} \approx 1\right)$. The comparison of the classical (stimulated) process described by Eq. (60) with the quantum (spontaneous) process described by $\overline{\mathrm{Eq}}$. (58) largely follows the comparison between Eqs. (51) and (하), respectively, for second-order processes. Again we find that the generation bandwidth time $\mathcal{T}$ can be calculated, with the result here given by

$$
\mathcal{T}=\frac{2 L}{v_{\mu_{P}}\left|F_{\mu_{P}}\left(\omega_{\mu_{P}}\right)\right|^{2}}=\frac{\omega_{\mu_{P}} L^{2}}{2 v_{\mu_{P}}^{2} Q_{\mu_{P}}},
$$

and thus, if photons are collected across an entire ring resonance linewidth, substituting Eq. (타) in Eq. (다), we find

$$
\begin{aligned}
P_{I} & =\frac{\hbar \omega_{\mu_{P}} v_{\mu_{P}}}{2 L\left|F_{\mu_{P}}\left(\omega_{\mu_{P}}\right)\right|^{2}}\left(\gamma P_{P} L\right)^{2}\left|F_{\mu_{P}}\left(\omega_{\mu_{P}}\right)\right|^{8} \\
& =\frac{\hbar \omega_{\mu_{P}}}{\frac{Q_{\mu_{P}}}{64 \pi} \tau_{\mu_{P}}}\left(\gamma P_{P} L\right)^{2}\left(\frac{Q_{\mu_{P}} v_{\mu_{P}}}{\omega_{\mu_{P}} L}\right)^{4}
\end{aligned}
$$

where we have introduced $\tau_{\mu_{P}}=2 \pi / \omega_{\mu_{P}}$. Note that the appearance of $Q_{\mu_{P}}$ in the generation bandwidth time leads to a net dependence of $P_{I}$ on three powers of $Q_{\mu_{P}}$, rather than the four that might be expected from Eq. (60). If one had a filter with bandwidth $2 \pi B$, and collected photons generated within just a small window of a ring resonance, one would find instead

$P_{I}(\Omega)=\frac{\hbar \omega_{\mu_{P}}}{B^{-1}}\left(\gamma P_{P} L\right)^{2}\left|F_{\mu_{P}}\left(\omega_{\mu_{P}}\right)\right|^{4}\left|F_{\mu_{P}}\left(\omega_{\mu_{P}}-\Omega\right)\right|^{2}\left|F_{\mu_{P}}\left(\omega_{\mu_{P}}+\Omega\right)\right|^{2}$,

where $\Omega+\omega_{\mu_{P}}$ is the center of the narrow frequency window selected by the filter.

Once more there is an entire frequency comb of generated photons. Here the generation rate is reduced by a factor of 2 when $\quad \omega_{\mu_{S}}+\omega_{\mu_{I}}-2 \omega_{\mu_{P}}=4 v_{\mu_{P}} /\left(\left|F_{\mu_{P}}\left(\omega_{\mu_{P}}\right)\right|^{2} L\right)=\omega_{\mu_{P}} / Q_{\mu_{P}}$. Taylor expanding as above while remembering that if $\omega_{\mu_{S}}$ is associated with resonance order $N$ that $\omega_{\mu_{I}}$ is associated with resonance order $2 N_{P}-N$, we find that, quite similar to above, when

$$
N-N_{P}=R\left[\omega_{\mu_{P}} /\left(2\left|\Xi_{P}\right| Q_{\mu_{P}}\right)\right]^{1 / 2},
$$

the generation rate drops by a factor of 2 .

We conclude our analysis by summarizing in Table $\underline{2}$ all of the results for stimulated and spontaneous processes in microring resonators. Here we report the "idler" powers as a function of the quality factors $Q_{\mu}$ and ring radius $R$, which are parameters that can be easily measured and thus are experimentally used to characterize a device. These quantities are directly related to the on-resonant intensity enhancement factors $\left|F_{\mu_{P}}\left(\omega_{\mu_{P}}\right)\right|^{2}$, as such enhancement depends on the effective mode volume $(\propto \mathcal{A} R)$ as well as constructive interference of the field in the ring, which is proportional to the time that light spends in the resonator (proportional to $Q$ ).
Looking at the expressions reported in Table 2 , we see that for DFG and SPDC, the generated powers are inversely proportional to $\mathcal{A} R$. Similarly, the generated idler powers in FWM and SFWM scale with the inverse of $(\mathcal{A} R)^{2}$. In other words, the intensity enhancement associated with the volume of the region in which light is confined has the same weight, whether the process is spontaneous or stimulated. The situation is different if we focus on the scaling of the generated powers with $Q$. In this case, unfiltered SPDC and SFWM "idler" powers are proportional to $Q^{2}$ and $Q^{3}$, respectively, while the corresponding processes DFG and FWM scale with $Q^{3}$ and $Q^{4}$, respectively. Finally, as in channel waveguides, stimulated and spontaneous powers scale the same when we consider the spontaneous generated power in a sufficiently narrow spectral region centered at the idler/signal resonance.

\section{CONCLUSION}

Integrated nonlinear optical devices for use in the classical regime are under constant development and improvement. Many of these hold potential for use in quantum optics. Yet given the calculated or measured efficiency of such a device in the classical regime, it is not always easy to predict its efficiency in the quantum regime. In general, the power generated by a spontaneous process in a given device does not scale with device parameters in the same way as the power generated by a stimulated process. Here we have employed a general Hamiltonian formalism, which places quantum and classical wave mixing processes on equal theoretical footing, to calculate expressions for SPDC, DFG, SHG, SFWM, and FWM in both channel waveguides and microring resonators side-coupled to channel waveguides as two examples of devices. These expressions are presented as two comprehensive tables that clearly answer the questions posed in the introduction. In channel waveguides, the scaling behavior of both stimulated and spontaneous processes with the length $L$ of the channel is independent of the order of the nonlinearity. In particular, the efficiency of phase-matched stimulated generation scales as $L^{2}$, whether we are considering DFG or FWM. The same scaling is obtained for the generation rate of spontaneous processes within a sufficiently narrow spectral region around the phase-matching condition. Similarly, the generated idler power, integrated over the entire spectrum, scales with $L^{3 / 2}$ for both SPDC and SFWM in a channel, as in the absence of filtering the generation bandwidth time scales as $L^{1 / 2}$. This is in contrast to the results for a microring resonator, in which all second-order processes scale with ring radius $R$ as $R^{-1}$ and all third-order processes as $R^{-2}$ regardless of filtering. Considering enhancement due to light confinement in the channel, which is inversely proportional to the effective area, we observe that this effect on the generated power is independent of the nature of the process, stimulated or spontaneous, while it depends upon the order of the nonlinearity. Indeed, for DFG and SPDC, the generation rate is proportional to $1 / \mathcal{A}$, while for FWM and SFWM, it scales with $\gamma^{2} \propto 1 / \mathcal{A}^{2}$. This result is consistent with the fact that for the example of a channel the scaling with $L$ is due solely to the interference of the fields generated at different positions in the channel. That is, there is no $L$-dependent field enhancement. Again this is in contrast with the results for a microring side-coupled to a channel waveguide, in which the additional confinement in the direction of propagation due to the ring plays a role. DFG and FWM 
scale with $Q^{3}$ and $Q^{4}$, respectively, in a ring resonator as do the corresponding spontaneous processes within a sufficiently narrow spectral region centered at the idler/signal resonance. Unfiltered SPDC and SFWM generated powers, on the other hand, are proportional to $Q^{2}$ and $Q^{3}$, respectively.

\section{APPENDIX A}

We begin with the Hamiltonian (28), where

$$
\begin{aligned}
S\left(k_{1}, k_{2}, k_{3}, k_{4}\right)= & \frac{3}{2} \sqrt{\frac{\left(\hbar \omega_{k_{1}}\right)\left(\hbar \omega_{k_{2}}\right)\left(\hbar \omega_{k_{3}}\right)\left(\hbar \omega_{k_{4}}\right)}{(4 \pi)^{4} \varepsilon_{0}^{2}}} \frac{\bar{\chi}_{3}}{\bar{n}^{4}} \\
& \int_{-L / 2}^{L / 2} \mathrm{~d} z e^{i\left(k_{3}+k_{4}-k_{1}-k_{2}\right) z} \frac{e^{i \phi\left(k_{1}, k_{2}, k_{3}, k_{4}\right)}}{\mathcal{A}\left(k_{1}, k_{2}, k_{3}, k_{4}\right)},
\end{aligned}
$$

and

$$
i \hbar \frac{\partial \overline{\mathcal{O}}(t)}{\partial t}=[\overline{\mathcal{O}}(t), \hat{V}(t)]
$$

For a coherent asymptotic-in state

$$
\left|\psi_{\text {in }}\right\rangle=e^{\left(\alpha A_{P}^{\dagger}-\text { H.c. }\right)}|\operatorname{vac}\rangle
$$

with an average photon number of $\mathcal{N}_{P}=|\alpha|^{2}$, where

$$
A_{P}^{\dagger}=\int \mathrm{d} k \phi_{P}(k) a_{k}^{\dagger}
$$

and

$$
\int \mathrm{d} k\left|\phi_{P}(k)\right|^{2}=1
$$

$$
\frac{e^{i \phi\left(k_{1}, k_{2}, k_{3}, k_{4}\right)}}{\mathcal{A}\left(k_{1}, k_{2}, k_{3}, k_{4}\right)}=\int \mathrm{d} x \mathrm{~d} y \frac{\bar{n}^{4} \chi_{3}^{i j k l}(x, y)\left[d_{k_{1}}^{i}(x, y)\right]^{*}\left[d_{k_{2}}^{j}(x, y)\right]^{*} d_{k_{3}}^{k}(x, y) d_{k_{4}}^{l}(x, y)}{\bar{\chi}_{3} \varepsilon_{0}^{2} n^{2}\left(x, y ; \omega_{k_{1}}\right) n^{2}\left(x, y ; \omega_{k_{2}}\right) n^{2}\left(x, y ; \omega_{k_{3}}\right) n^{2}\left(x, y ; \omega_{k_{4}}\right)}
$$

is an effective area with $\chi_{3}^{i j k l}(x, y)$ the material third-order optical nonlinearity neglecting dispersion and magneto-optic effects, $d_{\omega}^{i}(x, y)$ the $i$ th component of the displacement field at frequency $\omega$, and $n(x, y ; \omega)$ the material refractive index at frequency $\omega$, all at waveguide cross-sectional position $(x, y)$. Following the backward Heisenberg picture approach of [9], we then split the Hamiltonian into a trivial evolution piece

$$
H_{0}=H_{L}=\int \mathrm{d} k \hbar \omega_{k} a_{k}^{\dagger} a_{k}
$$

and an appropriately transformed time-dependent nonlinear evolution piece

$$
\begin{aligned}
\hat{V}(t) & =U\left(t_{1}, t\right) e^{i H_{0} t / \hbar} H_{N L} e^{-i H_{0} t / \hbar} U^{\dagger}\left(t_{1}, t\right) \\
& =-\int \mathrm{d} k_{1} \mathrm{~d} k_{2} \mathrm{~d} k_{3} \mathrm{~d} k_{4} S\left(k_{1}, k_{2}, k_{3}, k_{4} ; t\right) \bar{a}_{k_{1}}^{\dagger}(t) \bar{a}_{k_{2}}^{\dagger}(t) \bar{a}_{k_{3}}(t) \bar{a}_{k_{4}}(t),
\end{aligned}
$$

where

$$
\begin{gathered}
U\left(t^{\prime}, t\right)=e^{i H_{0} t^{\prime} / \hbar} e^{-i H\left(t^{\prime}-t\right) / \hbar} e^{-i H_{0} t / \hbar}, \\
\overline{\mathcal{O}}(t)=U\left(t_{1}, t\right) \mathcal{O} U^{\dagger}\left(t_{1}, t\right),
\end{gathered}
$$

with

$$
\overline{\mathcal{O}}\left(t_{1}\right)=\mathcal{O}
$$

and

$$
S\left(k_{1}, k_{2}, k_{3}, k_{4} ; t\right)=S\left(k_{1}, k_{2}, k_{3}, k_{4}\right) e^{i\left(\omega_{k_{1}}+\omega_{k_{2}}-\omega_{k_{3}}-\omega_{k_{4}}\right) t},
$$

such that the barred operators satisfy the equations of motion the asymptotic-out state can be written

$$
\left|\psi_{\text {out }}\right\rangle=e^{\alpha \bar{A}_{P}^{\dagger}\left(t_{0}\right)-\text { H.c. }}|\mathrm{vac}\rangle
$$

where

$$
\bar{A}_{P}^{\dagger}\left(t_{0}\right)=\int \mathrm{d} k \phi_{P}(k) \bar{a}_{k}^{\dagger}\left(t_{0}\right)
$$

and [recall (A9)] the barred operator satisfies

$$
i \hbar \frac{d \bar{a}_{k}^{\dagger}(t)}{d t}=2 \int \mathrm{d} k_{1} \mathrm{~d} k_{2} \mathrm{~d} k_{3} S\left(k_{1}, k_{2}, k_{3}, k ; t\right) \bar{a}_{k_{1}}^{\dagger}(t) \bar{a}_{k_{2}}^{\dagger}(t) \bar{a}_{k_{3}}(t) .
$$

This equation has the zeroth-order solution

$$
\left(\bar{a}_{k}^{\dagger}(t)\right)^{0}=\bar{a}_{k}^{\dagger}\left(t_{1}\right)=a_{k}^{\dagger}
$$

and so the first-order solution for the operator involved in our asymptotic-out state is

$$
\begin{aligned}
\bar{a}_{k}^{\dagger}(t)= & a_{k}^{\dagger}+\frac{2}{i \hbar} \int \mathrm{d} k_{1} \mathrm{~d} k_{2} \mathrm{~d} k_{3}\left[\int_{t_{1}}^{t_{0}} \mathrm{~d} t S\left(k_{1}, k_{2}, k_{3}, k ; t\right)\right] a_{k_{1}}^{\dagger} a_{k_{2}}^{\dagger} a_{k_{3}} \\
= & a_{k}^{\dagger}+\frac{2 i}{\hbar} \int \mathrm{d} k_{1} \mathrm{~d} k_{2} \mathrm{~d} k_{3}\left[\int_{t_{0}}^{t_{1}} \mathrm{~d} t S\left(k_{1}, k_{2}, k_{3}, k ; t\right)\right] a_{k_{1}}^{\dagger} a_{k_{2}}^{\dagger} a_{k_{3}} \\
= & a_{k}^{\dagger}+\frac{4 \pi i}{\hbar} \int \mathrm{d} k_{1} \mathrm{~d} k_{2} \mathrm{~d} k_{3} S\left(k_{1}, k_{2}, k_{3}, k\right) \\
& a_{k_{1}}^{\dagger} a_{k_{2}}^{\dagger} a_{k_{3}} \delta\left(\omega_{k_{1}}+\omega_{k_{2}}-\omega_{k_{3}}-\omega_{k}\right),
\end{aligned}
$$

where we have extended the range of integration from $t_{0} \rightarrow$ $-\infty$ to $t_{1} \rightarrow \infty$. An important subtlety is that the state of generated photons in the $\chi_{3}$ process contains noncommuting operators and cannot readily be split into an undepleted pump 
field and a generated field as in the case of a $\chi_{2}$ process. This is in part due to the fact that we do not divide $k$ (or $\omega$ ) space up into two regions as in the case of a $\chi_{2}$ process-we assume that there is just one mode to consider, and thus no additional labels appear. To get around this problem, when writing down the state of generated photons for a SFWM process, we use the Baker-Campbell-Hausdorff formula [24] to keep only leading-order terms in the exponential that involve twophoton creation operators. Thus, following

$$
e^{A+B}=e^{A} e^{-\frac{1}{2}[A, B]+\text { higher order terms }} e^{B},
$$

where any one of the "higher order terms" is proportional to

$$
\left[S_{1},\left[S_{2}, \cdots\left[S_{n-1}, S_{n}\right] \cdots\right]\right], S_{i=1,2, \cdots n}=A \quad \text { or } \quad B
$$

we take

$A=\alpha \int \mathrm{d} k \phi_{P}(k) a_{k}^{\dagger}-$ H.c.,

$B=\frac{4 \pi i \alpha}{\hbar} \int \mathrm{d} k \mathrm{~d} k_{1} \mathrm{~d} k_{2} \mathrm{~d} k_{3} \phi_{P}(k) S\left(k_{1}, k_{2}, k_{3}, k\right) a_{k_{1}}^{\dagger} a_{k_{2}}^{\dagger} a_{k_{3}}$ - H.c.,

and work out

$$
\begin{aligned}
-\frac{1}{2}[A, B]= & \frac{2 \pi i \alpha^{2}}{\hbar} \int \mathrm{d} k \mathrm{~d} k_{1} \mathrm{~d} k_{2} \mathrm{~d} k_{3} \phi_{P}\left(k_{3}\right) \phi_{P}(k) \\
& \times S\left(k_{1}, k_{2}, k_{3}, k\right) a_{k_{1}}^{\dagger} a_{k_{2}}^{\dagger}-\text { H.c. } \\
& +\frac{8 \pi i|\alpha|^{2}}{\hbar} \int \mathrm{d} k \mathrm{~d} k_{1} \mathrm{~d} k_{2} \mathrm{~d} k_{3} \phi_{P}^{*}\left(k_{2}\right) \phi_{P}(k) \\
& \times S\left(k_{1}, k_{2}, k_{3}, k\right) a_{k_{1}}^{\dagger} a_{k_{3}}-\text { H.c.. }
\end{aligned}
$$

Note that with the definition above Eq. (19),

$$
e^{B}|\mathrm{vac}\rangle=|\mathrm{vac}\rangle
$$

and thus we may write our asymptotic-out state, neglecting all higher-order and non-two-photon creation operator terms mentioned above, as

$$
\left|\psi_{\text {out }}\right\rangle=e^{\left(\alpha A_{P}^{\dagger}+\beta C_{\mathrm{II}}^{\dagger}\right)-\text { H.c. }}|\operatorname{vac}\rangle,
$$

where

$$
\begin{gathered}
C_{\mathrm{II}}^{\dagger}=\frac{1}{\sqrt{2}} \int \mathrm{d} k_{1} \mathrm{~d} k_{2} \phi\left(k_{1}, k_{2}\right) a_{k_{1}}^{\dagger} a_{k_{2}}^{\dagger}, \\
\phi\left(k_{1}, k_{2}\right)=\frac{2 \sqrt{2} \pi \alpha^{2}}{\beta} \frac{i}{\hbar} \int \mathrm{d} k \mathrm{~d} k^{\prime} \phi_{P}(k) \phi_{P_{3}}\left(k^{\prime}\right) \\
\times S\left(k_{1}, k_{2}, k, k^{\prime}\right) \delta\left(\omega_{k}+\omega_{k^{\prime}}-\omega_{k_{1}}-\omega_{k_{2}}\right),
\end{gathered}
$$

and $\beta$ is chosen such that the biphoton wave function is normalized

$$
\int \mathrm{d} k_{1} \mathrm{~d} k_{2}\left|\phi\left(k_{1}, k_{2}\right)\right|^{2}=1
$$

Note that for $|\beta| \ll 1$, we may write the state of generated photons as

$$
\left|\psi_{\text {gen }}\right\rangle=e^{\beta C_{\mathrm{II}}^{\dagger}-\text { H.c. }}|\operatorname{vac}\rangle \approx|\operatorname{vac}\rangle+\beta|\mathrm{II}\rangle+\cdots,
$$

where

$$
|\mathrm{II}\rangle=C_{\mathrm{II}}^{\dagger}|\mathrm{vac}\rangle=\frac{1}{\sqrt{2}} \int \mathrm{d} k_{1} \mathrm{~d} k_{2} \phi\left(k_{1}, k_{2}\right) a_{k_{1}}^{\dagger} a_{k_{2}}^{\dagger}|\mathrm{vac}\rangle
$$

is a normalized two-photon state

$$
\int \mathrm{d} k a_{k}^{\dagger} a_{k}|\mathrm{II}\rangle=2|\mathrm{II}\rangle,\langle\mathrm{II} . \mid \mathrm{II}\rangle=1
$$

and therefore identify $|\beta|^{2}$ with the probability of pair production, making the average number of generated photon pairs per pump pulse

$$
\mathcal{N}_{D}=2|\beta|^{2}
$$

If we assume that the pump waveform $\phi_{P}(k)$ is peaked strongly enough for some $k_{0}>0$ such that its values are negligible for $k<0$, the integrals over $k$ involved in the biphoton wave function may be restricted to the range 0 to $\infty$, and this in turn, due to the energy conserving delta function and approximate momentum conserving $S$ function, means that the biphoton wave function itself will be nonnegligible only for $k_{1}>0$ and $k_{2}>0$ if typical dispersion relations are assumed. This creates a one-to-one correspondence between wavenumbers and frequencies, and so we may write expressions in the frequency representation by introducing

$$
\tilde{a}_{\omega}=\sqrt{\frac{d k(\omega)}{d \omega}} a_{k(\omega)}
$$

$$
\tilde{\phi}_{P}(\omega)=\sqrt{\frac{d k(\omega)}{d \omega}} \phi_{P}(k(\omega)),
$$

$$
\tilde{\phi}\left(\omega_{1}, \omega_{2}\right)=\sqrt{\frac{d k\left(\omega_{1}\right)}{d \omega_{1}}} \sqrt{\frac{d k\left(\omega_{2}\right)}{d \omega_{2}}} \phi\left(k\left(\omega_{1}\right), k\left(\omega_{2}\right)\right),
$$

where

$$
\frac{d k\left(\omega_{1}\right)}{d \omega_{1}}=\left(\frac{d k(\omega)}{d \omega}\right)_{\omega=\omega_{1}},
$$

and the prefactors ensure correct normalization

$$
\int_{0}^{\infty} \mathrm{d} \omega\left|\tilde{\phi}_{P}(\omega)\right|^{2}=1
$$

$$
\int_{0}^{\infty} \mathrm{d} \omega_{1} \int_{0}^{\infty} \mathrm{d} \omega_{2}\left|\tilde{\phi}\left(\omega_{1}, \omega_{2}\right)\right|^{2}=1
$$

This allows us to write the biphoton wave function as 


$$
\begin{aligned}
\tilde{\phi}\left(\omega_{1}, \omega_{2}\right)= & \frac{2 \sqrt{2} \pi \alpha^{2}}{\beta} \frac{i}{\hbar} \sqrt{\frac{d k\left(\omega_{1}\right)}{d \omega_{1}}} \sqrt{\frac{d k\left(\omega_{2}\right)}{d \omega_{2}}} \int_{0}^{\infty} \mathrm{d} \omega \int_{0}^{\infty} \mathrm{d} \omega^{\prime} \\
& \left\{\sqrt{\frac{d k(\omega)}{d \omega}} \sqrt{\frac{d k\left(\omega^{\prime}\right)}{d \omega^{\prime}}} \phi_{P}(\omega) \phi_{P}\left(\omega^{\prime}\right)\right. \\
& \left.S\left[k\left(\omega_{1}\right), k\left(\omega_{2}\right), k(\omega), k\left(\omega^{\prime}\right)\right] \delta\left(\omega_{1}+\omega_{2}-\omega-\omega^{\prime}\right)\right\},
\end{aligned}
$$

and use Eq. (A35) to solve for the normalization factor

$$
\mathcal{N}_{D}=\frac{\mathcal{N}_{P}^{2}\left(\bar{\chi}_{3}\right)^{2}}{\bar{n}^{8}} \frac{9 K}{64 \pi^{2} \varepsilon_{0}^{2}},
$$

where

$$
K=\int_{0}^{\infty} \omega_{1} \frac{d k\left(\omega_{1}\right)}{d \omega_{1}} \int_{0}^{\infty} \omega_{2} \frac{d k\left(\omega_{2}\right)}{d \omega_{2}} J\left(\omega_{1}, \omega_{2}\right) \mathrm{d} \omega_{1} \mathrm{~d} \omega_{2}
$$

with

$$
\begin{aligned}
J\left(\omega_{1}, \omega_{2}\right)= & \mid \int_{0}^{\omega_{1}+\omega_{2}} \mathrm{~d} \omega \sqrt{\frac{d k(\omega)}{d \omega}} \sqrt{\frac{d k\left(\omega_{1}+\omega_{2}-\omega\right)}{d\left(\omega_{1}+\omega_{2}-\omega\right)}} \tilde{\phi}_{P}(\omega) \\
& \times \tilde{\phi}_{P}\left(\omega_{1}+\omega_{2}-\omega\right) \hbar \sqrt{\omega\left(\omega_{1}+\omega_{2}-\omega\right)} \\
& \times \int_{-L / 2}^{L / 2} \mathrm{~d} z e^{i\left(k(\omega)+k\left(\omega_{1}+\omega_{2}-\omega\right)-k\left(\omega_{1}\right)-k\left(\omega_{2}\right)\right) z} \\
& \times\left.\frac{e^{i \phi\left(k\left(\omega_{1}\right), k\left(\omega_{2}\right), k(\omega), k\left(\omega_{1}+\omega_{2}-\omega\right)\right)}}{\mathcal{A}\left[k\left(\omega_{1}\right), k\left(\omega_{2}\right), k(\omega), k\left(\omega_{1}+\omega_{2}-\omega\right)\right]}\right|^{2} .
\end{aligned}
$$

We assume that the derivatives $d k(\omega) / d \omega$ change very slowly with $\omega$, and so approximate them as constants $d k(\omega) / d \omega \approx 1 / v$ with little error. Similarly, we assume the effective area to be constant over the frequencies of interest and write $\mathcal{A}(k(\omega)$, $\left.k\left(\omega_{1}+\omega_{2}-\omega\right), k\left(\omega_{1}\right), k\left(\omega_{2}\right)\right) \approx \mathcal{A}$. Furthermore it is possible to define the mode amplitudes such that we can take the phase $\phi\left(k(\omega), k\left(\omega_{1}+\omega_{2}-\omega\right), k\left(\omega_{1}\right), k\left(\omega_{2}\right)\right)$ to be zero. We specify the dispersion of the waveguide using a quadratic model around the phase-matching condition

$$
k(\omega)=k\left(\omega_{P}\right)+\frac{1}{v}\left(\omega-\omega_{P}\right)+\frac{\beta_{2}\left(\omega_{P}\right)}{2}\left(\omega-\omega_{P}\right)^{2},
$$

where $v^{-1}=(d k(\omega) / d \omega)_{\omega=\omega_{P}}, \quad \beta_{2}\left(\omega_{P}\right)=\left(d^{2} k(\omega) / d \omega^{2}\right)_{\omega=\omega_{P}}$, and find

$$
\begin{aligned}
\tilde{\phi}\left(\omega_{1}, \omega_{2}\right)= & i \frac{L}{v^{2}} \sqrt{\frac{\hbar^{2} \omega_{1} \omega_{2}}{K \mathcal{A}^{2}}} \int_{0}^{\omega_{1}+\omega_{2}} \mathrm{~d} \omega \frac{\sin \left[\left(\omega-\omega_{P}\right) \Delta_{t} / 2\right]}{\left(\omega-\omega_{P}\right) \sqrt{\pi \Delta_{t} / 2}} \\
& \frac{\sin \left[\left(\omega_{1}+\omega_{2}-\omega-\omega_{P}\right) \Delta_{t} / 2\right]}{\left(\omega_{1}+\omega_{2}-\omega-\omega_{P}\right) \sqrt{\pi \Delta_{t} / 2}} \\
& \times \sqrt{\omega\left(\omega_{1}+\omega_{2}-\omega\right)} \operatorname{sinc}\left\{\frac { \beta _ { 2 } ( \omega _ { P } ) } { 2 } \left[\left(\omega-\frac{\omega_{1}+\omega_{2}}{2}\right)^{2}\right.\right. \\
& \left.\left.-\left(\frac{\omega_{1}-\omega_{2}}{2}\right)^{2}\right] L\right\},
\end{aligned}
$$

$$
\begin{aligned}
K= & \frac{\hbar^{2} L^{2}}{v^{4} \mathcal{A}^{2}} \int_{0}^{\infty} \mathrm{d} \omega_{1} \int_{0}^{\infty} \mathrm{d} \omega_{2} \omega_{1} \omega_{2} \mid \int_{0}^{\omega_{1}+\omega_{2}} \mathrm{~d} \omega \frac{\sin \left[\left(\omega-\omega_{P}\right) \Delta_{t} / 2\right]}{\left(\omega-\omega_{P}\right) \sqrt{\pi \Delta_{t} / 2}} \\
& \times \frac{\sin \left[\left(\omega_{1}+\omega_{2}-\omega-\omega_{P}\right) \Delta_{t} / 2\right]}{\left(\omega_{1}+\omega_{2}-\omega-\omega_{P}\right) \sqrt{\pi \Delta_{t} / 2}} \sqrt{\omega\left(\omega_{1}+\omega_{2}-\omega\right)} \\
& \times\left.\operatorname{sinc}\left\{\frac{\beta_{2}\left(\omega_{P}\right)}{2}\left[\left(\omega-\frac{\omega_{1}+\omega_{2}}{2}\right)^{2}-\left(\frac{\omega_{1}-\omega_{2}}{2}\right)^{2}\right] L\right\}\right|^{2},(\mathrm{~A} 42)
\end{aligned}
$$

where, as we have the CW limit in mind, we have taken as our pump waveforms "top-hat" pulses in time, that have the shape of sinc functions in frequency, centered at $\omega_{P}$, and will later take the limit of an infinite width in time

$$
\tilde{\phi}_{P}(\omega)=\frac{\sin \left[\left(\omega-\omega_{P}\right) \Delta_{t} / 2\right]}{\left(\omega-\omega_{P}\right) \sqrt{\pi \Delta_{t} / 2}}
$$

Making the change of variables $\omega_{t}=\omega_{1}+\omega_{2}$, $\omega_{r}=\left(\omega_{1}-\omega_{2}\right) / 2$, extending the integral over $\omega$ from $-\infty \rightarrow \infty$, and setting $\omega \approx \omega_{P}, \omega_{t} \approx 2 \omega_{P}$ (a good approximation in the $\Delta_{t} \rightarrow \infty$ limit), we find

$$
\begin{aligned}
K= & \frac{4 \hbar^{2} L^{2} \omega_{P}^{2} \pi}{v^{4} \mathcal{A}^{2} \Delta_{t}} \int_{0}^{\infty} \mathrm{d} \omega_{t} \delta\left(\omega_{t}\right. \\
& \left.-2 \omega_{P}\right) \int_{0}^{\frac{1}{2} \omega_{t}} \mathrm{~d} \omega_{r}\left(\frac{1}{4} \omega_{t}^{2}-\omega_{r}^{2}\right) \operatorname{sinc}^{2}\left(\beta_{2}\left(\omega_{P}\right) \omega_{r}^{2} L / 2\right) \\
= & \frac{4 \hbar^{2} L^{2} \omega_{P}^{2} \pi}{v^{4} \mathcal{A}^{2} \Delta_{t}} \int_{0}^{\omega_{P}} \mathrm{~d} \omega_{r}\left(\omega_{P}^{2}-\omega_{r}^{2}\right) \operatorname{sinc}^{2}\left(\beta_{2}\left(\omega_{P}\right) \omega_{r}^{2} L / 2\right) \\
= & \frac{8 \hbar^{2} L^{2} \omega_{P}^{4} \pi^{2}}{v^{4} \mathcal{A}^{2} \Delta_{t} \mathcal{T}},
\end{aligned}
$$

where $\mathcal{T}$ is the generation bandwidth time defined earlier [Eq. (32)]. Thus, recalling Eq. (A37) and introducing the nonlinear parameter [15]

$$
\gamma=\frac{3 \bar{\chi}_{3} \omega_{P}}{4 \varepsilon_{0} v^{2} \bar{n}^{4} \mathcal{A}},
$$

the number of photons per pump pulse can be written

$$
\mathcal{N}_{D}=\frac{2\left(\gamma \hbar \omega_{P} \mathcal{N}_{P} L\right)^{2}}{\Delta_{t} \mathcal{I}}
$$

Yet it is also true that if we consider a constant rate of pump photons or pump power, $P_{P}=\hbar \omega_{P} \mathcal{N}_{P} / \Delta_{t}, \quad \mathcal{N}_{D} / \Delta_{t}=$ $2\left(\gamma \hbar \omega_{P} \mathcal{N}_{P} L\right)^{2} /\left(\Delta_{t}^{2} \mathcal{T}\right)$ can be thought of as the average rate of generated photons provided that $|\beta| \sqrt{p_{\lambda}} \ll 1$, where $p_{\lambda}$ is the largest Schmidt coefficient in a Schmidt decomposition [12] of the biphoton wave function. Thus Eq. (A37) remains true in the CW limit, provided $P_{P} \ll(\gamma L)^{-1}$. Defining $P_{I}=$ $\hbar \omega_{P} \mathcal{N}_{D} /\left(2 \Delta_{t}\right)$ as the average generated power associated with one photon of each pair, we rewrite Eq. (A37) in a form suggestive of the corresponding stimulated result as

$$
P_{I}=\frac{\hbar \omega_{P}}{\mathcal{T}}\left(\gamma P_{P} L\right)^{2}
$$

which is the same as the result presented above [Eq. (31)]. 


\section{APPENDIX B}

We begin this section as the previous appendix, with the Hamiltonian (28), and terms defined as above [Eqs. (스) and (A2)]. Coupled mode equations are derived by Taylor expanding dispersion relations

$$
\omega_{m k}=\omega_{m}+v_{m}\left(k-k_{m}\right)+\ldots,
$$

where

$$
\begin{gathered}
\omega_{m}=\left(\omega_{m k}\right)_{k=k_{m}}, \\
v_{m}=\left(\frac{\partial \omega_{m k}}{\partial k}\right)_{k=k_{m}}
\end{gathered}
$$

in the linear regime, and as a first approximation, setting

$$
\begin{gathered}
\omega_{m k} \approx \omega_{m}, \\
d_{m k}^{i}(x, y) \approx d_{m}^{i}(x, y)
\end{gathered}
$$

in the nonlinear terms, and introducing effective fields

$$
g_{m}(z, t)=\int \frac{\mathrm{d} k}{\sqrt{2 \pi}} a_{m k} e^{i\left(k-k_{m}\right) z},
$$

where the integral goes over the range of $k$ space appropriate for the mode of interest. Note that while the pump, signal, and idler may or may not exist in distinct propagation modes of the waveguide, their center frequencies may be separated enough that we may still define a $g_{m}(z, t)$ for each. Indeed because of this separation, the points at which the $k$ 's appearing in the $g_{m}(z, t)$ are most naturally expanded about may be wellseparated, and as such the ranges over which each $e^{i\left(k-k_{m}\right) z}$ is significantly nonzero will also be well-separated, meaning we can introduce three $m$ labels ( $m=P$ for pump, $m=S$ for signal, $m=I$ for idler) corresponding to what formerly had no label at all, with

$$
\left[g_{S}(z, t), g_{I}^{\dagger}(z, t)\right]=\left[g_{S}(z, t), g_{P}^{\dagger}(z, t)\right]=\left[g_{I}(z, t), g_{P}^{\dagger}(z, t)\right]=0 .
$$

This is most certainly the case for the CW process that we consider here. We thus rewrite our initial Hamiltonian (28) as

$$
\begin{aligned}
H_{L}= & \sum_{m=P, S, I}\left[\hbar \omega_{m} \int \mathrm{d} z g_{m}^{\dagger} g_{m}+\frac{i}{2} \hbar v_{m}\right. \\
& \left.\int \mathrm{d} z\left(\frac{\partial g_{m}^{\dagger}}{\partial z} g_{m}-g_{m}^{\dagger} \frac{\partial g_{m}}{\partial z}\right)\right], \\
H_{N L}= & -\frac{3}{\varepsilon_{0}} \sqrt{\frac{\left(\hbar \omega_{P}\right)^{2}\left(\hbar \omega_{S}\right)\left(\hbar \omega_{I}\right)}{2^{4} \mathcal{A}^{2}}} \frac{\bar{\chi}_{3}}{\bar{n}^{4}} \\
& \times \int \mathrm{d} z e^{i\left(2 k_{P}-k_{S}-k_{I}\right) z} g_{S}^{\dagger} g_{I}^{\dagger} g_{P}^{2}+\text { H.c. }
\end{aligned}
$$

as there are now 12 , and not 6 , ways to arrange the operators, we have neglected terms corresponding to self- and crossphase modulation, and

$$
\mathcal{A}=\left|\int \mathrm{d} x \mathrm{~d} y \frac{\bar{n}^{4} \chi_{3}^{i j k l}(x, y)}{\bar{\chi}_{3}} \frac{\left[d_{S}^{i}(x, y)\right]^{*}\left[d_{I}^{j}(x, y)\right]^{*} d_{P}^{k}(x, y) d_{P}^{l}(x, y)}{\varepsilon_{0}^{2} n^{2}\left(x, y ; \omega_{S}\right) n^{2}\left(x, y ; \omega_{I}\right) n^{4}\left(x, y ; \omega_{P}\right)}\right|^{-1},
$$

where, as above, we have chosen the mode amplitudes such that we can take the phase associated with the effective area to be zero. The Heisenberg equations of motion yield

$$
\begin{aligned}
\frac{\partial g_{P}}{\partial t}= & -\frac{i}{\hbar}\left[g_{P}, H\right] \\
= & -i \omega_{P} g_{P}-v_{P} \frac{\partial g_{P}}{\partial z} \\
& -\frac{6 i}{\hbar \varepsilon_{0}} \sqrt{\frac{\left(\hbar \omega_{P}\right)^{2}\left(\hbar \omega_{S}\right)\left(\hbar \omega_{I}\right)}{2^{4} \mathcal{A}^{2}}} \frac{\bar{\chi}_{3}}{\bar{n}^{4}} e^{-i\left(2 k_{P}-k_{S}-k_{I}\right) z} g_{P}^{\dagger} g_{S} g_{I},
\end{aligned}
$$

$$
\begin{aligned}
& \frac{\partial g_{S}}{\partial t}=-\frac{i}{\hbar}\left[g_{S}, H_{3}\right] \\
&=-i \omega_{S} g_{S}-v_{S} \frac{\partial g_{S}}{\partial z} \\
&-\frac{3 i}{\hbar \varepsilon_{0}} \sqrt{\frac{\left(\hbar \omega_{P}\right)^{2}\left(\hbar \omega_{S}\right)\left(\hbar \omega_{I}\right)}{2^{4} \mathcal{A}^{2}}} \frac{\bar{\chi}_{3}}{\bar{n}^{4}} e^{i\left(2 k_{P}-k_{S}-k_{I}\right) z} g_{I}^{\dagger} g_{P}^{2}, \\
& \frac{\partial g_{I}}{\partial t=}-\frac{i}{\hbar}\left[g_{I}, H_{3}\right] \\
&=-i \omega_{I} g_{I}-v_{I} \frac{\partial g_{I}}{\partial z} \\
&-\frac{3 i}{\hbar \varepsilon_{0}} \sqrt{\frac{\left(\hbar \omega_{P}\right)^{2}\left(\hbar \omega_{S}\right)\left(\hbar \omega_{I}\right)}{2^{4} \mathcal{A}^{2}}} \frac{\bar{\chi}_{3}}{\bar{n}^{4}} e^{i\left(2 k_{P}-k_{S}-k_{I}\right) z} g_{S}^{\dagger} g_{P}^{2} .
\end{aligned}
$$

We then put

$$
g_{m}=\tilde{g}_{m} e^{-i \omega_{m} t},
$$

$$
2 \omega_{P}=\omega_{S}+\omega_{I}
$$

and write the equations above in terms of new operators

$$
G_{m}=\sqrt{\hbar \omega_{m} v_{m}} \tilde{g}_{m},
$$

such that $G_{m}^{\dagger} G_{m}=P_{m}$ has units of power

$$
\frac{1}{v_{P}} \frac{\partial G_{P}}{\partial t}+\frac{\partial G_{P}}{\partial z}=-\frac{6 i}{\varepsilon_{0}} \sqrt{\frac{\omega_{P}^{2}}{2^{4} \mathcal{A}^{2} v_{S} v_{I} v_{P}^{2}}} \frac{\bar{\chi}_{3}}{\bar{n}^{4}} e^{-i\left(2 k_{P}-k_{S}-k_{I}\right) z} G_{P}^{\dagger} G_{S} G_{I},
$$

$$
\frac{1}{v_{S}} \frac{\partial G_{S}}{\partial t}+\frac{\partial G_{S}}{\partial z}=-\frac{3 i}{\varepsilon_{0}} \sqrt{\frac{\omega_{S}^{2}}{2^{4} \mathcal{A}^{2} v_{S} v_{I} v_{P}^{2}}} \frac{\bar{\chi}_{3}}{\bar{n}^{4}} e^{i\left(2 k_{P}-k_{S}-k_{I}\right) z} g_{I}^{\dagger} g_{P}^{2},
$$




$$
\frac{1}{v_{I}} \frac{\partial G_{I}}{\partial t}+\frac{\partial G_{I}}{\partial z}=-\frac{3 i}{\varepsilon_{0}} \sqrt{\frac{\omega_{I}^{2}}{2^{4} \mathcal{A}^{2} v_{S} v_{I} v_{P}^{2}}} \bar{\chi}_{3} \bar{n}^{4} e^{i\left(2 k_{P}-k_{S}-k_{I}\right) z} G_{S}^{\dagger} G_{P}^{2}
$$

We work in the undepleted pump (and seed) approximation, in the limit of stationary fields, where the time derivatives vanish, and the strong pump and signal limit, $G_{P, S} \gg G_{I}$, with a uniform nonlinearity extending from $z=-\frac{L}{2}$ to $z=\frac{L}{2}$, leaving

$$
\begin{gathered}
\frac{\partial G_{P}}{\partial z}=0 \\
\frac{\partial G_{S}}{\partial z}=0 \\
G_{I}=-\frac{3 i}{\varepsilon_{0}} \sqrt{\frac{\omega_{I}^{2}}{2^{4} \mathcal{A}^{2} v_{S} v_{I} v_{P}^{2}}} \frac{\bar{\chi}_{3}}{\bar{n}^{4}} G_{S}^{\dagger} G_{P}^{2} L \operatorname{sinc}\left[\left(2 k_{P}-k_{S}-k_{I}\right) \frac{L}{2}\right]
\end{gathered}
$$

or, switching to the notation of Agrawal [15], where

$$
\gamma=\frac{n_{2} \omega_{0}}{c A_{\mathrm{eff}}}=\frac{3 \chi_{3}^{x x x x}}{4 \varepsilon_{0} c \bar{n}^{2}} \frac{\omega_{0}}{c A_{\mathrm{eff}}},
$$

or, in our notation with $\bar{n}=\sqrt[4]{n_{P}^{2} n_{S} n_{I}}, v_{m} \approx c / n_{m}$,

$$
\gamma=\frac{3 \omega_{I}}{4 \varepsilon_{0} v_{P} \sqrt{v_{S} v_{I}}} \frac{\bar{\chi}_{3}}{\bar{n}^{4}} \frac{1}{\mathcal{A}}
$$

we find

$$
G_{I}=-i \gamma G_{S}^{\dagger} G_{P}^{2} L \operatorname{sinc}\left[\left(2 k_{P}-k_{S}-k_{I}\right) L / 2\right]
$$

and thus

$$
P_{I}=P_{S}\left(\gamma P_{P} L\right)^{2} \operatorname{sinc}^{2}\left\{2 k_{P}\left(\omega_{P}\right)-k_{S}\left(\omega_{S}\right)-k_{I}\left(2 \omega_{P}-\omega_{S}\right) L / 2\right\},
$$

exactly as above [Eq. (33)].

\section{ACKNOWLEDGMENTS}

This work is supported by the Natural Science and Engineering Research Council of Canada, and by the Italian Ministry of University and Research, FIRB Contract No. RBFR08QIP5.

\section{REFERENCES}

1. P. A. Franken, A. E. Hill, C. W. Peters, and G. Weinreich, "Generation of optical harmonics," Phys. Rev. Lett. 7, 118-119 (1961).

2. C. Monat, A. Clark, C. Xiong, C. Grillet, G. Marshall, M. J. Steel, J. Li, L. O'Faolain, T. F. Krauss, and B. J. Eggleton, "Correlated photon-pair generation in an ultra-compact silicon photonic crystal waveguide," in CLEO:2011, Baltimore (2011), paper PDPC4.

3. S. Tanzilli, W. Tittel, H. De Riedmatten, H. Zbinden, P. Baldi, M. DeMicheli, D. B. Ostrowsky, and N. Gisin, "PPLN waveguide for quantum communication,” Eur. Phys. J. D 18, 155-160 (2002).
4. J. E. Sharping, K. F. Lee, M. A. Foster, A. C. Turner, B. S. Schmidt, M. Lipson, A. L. Gaeta, and P. Kumar, "Generation of correlated photons in nanoscale silicon waveguides," Opt. Express 14, 12388-12393 (2006).

5. M. B. Nasr, S. Carrasco1, B. E. A. Saleh, A. V. Sergienko, M. C. Teich, J. P. Torres, L. Torner, D. S. Hum, and M. M. Fejer, "Ultrabroadband biphotons generated via chirped quasi-phasematched optical parametric down-conversion,” Phys. Rev. Lett. 100, 183601 (2008).

6. E. Y. Zhu, Z. Tang, L. Qian, L. G. Helt, M. Liscidini, J. E. Sipe, C. Corbari, A. Canagasabey, M. Ibsen, and P. G. Kazansky, "Direct generation of polarization-entangled photon pairs in a poled fiber,” Phys. Rev. Lett. 108, 213902 (2012).

7. C. Liang, K. F. Lee, M. Medic, and P. Kumar, "Characterization of fiber-generated entangled photon pairs with superconducting single-photon detectors," Opt. Express 15, 1322-1327 (2007).

8. R. Horn, P. Abolghasem, B. J. Bijlani, D. Kang, A. S. Helmy, and G. Weihs, "Monolithic source of photon pairs," Phys Rev. Lett. 108, 153605 (2012).

9. Z. Yang, M. Liscidini, and J. E. Sipe, "Spontaneous parametric down-conversion in waveguides: a backward Heisenberg picture approach," Phys. Rev. A 77, 033808 (2008).

10. L. G. Helt, E. Y. Zhu, M. Liscidini, L. Qian, and J. E. Sipe, "Proposal for in-fiber generation of telecom-band polarizationentangled photon pairs using a periodically poled fiber," Opt. Lett. 34, 2138-2140 (2009).

11. Z. Yang, P. Chak, A. D. Bristow, H. M. van Driel, R. Iyer, J. S. Aitchison, A. L. Smirl, and J. E. Sipe, "Enhanced secondharmonic generation in AlGaAs microring resonators," Opt. Lett. 32, 826-828 (2007).

12. C. K. Law and J. H. Eberly, "Analysis and interpretation of high transverse entanglement in optical parametric down conversion,” Phys. Rev. Lett. 92, 127903 (2004).

13. A. Yariv, Quantum Electronics, 2nd ed. (Wiley, 1975).

14. A. De Rossi, V. Berger, M. Calligaro, G. Leo, V. Ortiz, and X Marcadet, "Parametric fluorescence in oxidized gallium arsenide waveguides," Appl. Phys. Lett. 79, 3758-3760 (2001).

15. G. P. Agrawal, Nonlinear Fiber Optics, 4th ed. (Elsevier, 2006).

16. E. Brainis, "Four-photon scattering in birefringent fibers," Phys. Rev. A 79, 023840 (2009).

17. C. Xiong, G. D. Marshall, A. Peruzzo, M. Lobino, A. S. Clark, D.-Y. Choi, S. J. Madden, C. M. Natarajan, M. G. Tanner, R. H. Hadfield, S. N. Dorenbox, T. Zijlstra, V. Zsiller, M. G. Thompson, J. G. Rarity, M. J. Steel, B. Luther-Davies, B. J. Eggleton, and J. L. O'Brien, "Generation of correlated photon pairs in a chalcogenide $\mathrm{As}_{2} \mathrm{~S}_{3}$ waveguide,” Appl. Phys. Lett. 98, 051101 (2011).

18. M. Ferrera, L. Razzari1, D. Duchesne, R. Morandotti, Z. Yang, M. Liscidini, J. E. Sipe, S. Chu, B. E. Little, and D. J. Moss, "Lowpower continuous-wave nonlinear optics in doped silica glass integrated waveguide structures," Nat. Photon. 2, 737-740 (2008).

19. A. C. Turner, M. A. Foster, A. L. Gaeta, and M. Lipson, "Ultra-low power parametric frequency conversion in a silicon microring resonator," Opt. Express 16, $4881-4887$ (2008).

20. J. E. Heebner, R. Grover, and T. Ibrahim, Optical Microresonators: Theory, Fabrication, and Applications (Springer, 2008).

21. Z. Yang and J. E. Sipe, "Generating entangled photons via enhanced spontaneous parametric downconversion in AlGaAs microring resonators," Opt. Lett. 32, 3296-3298 (2007).

22. P. P. Absil, J. V. Hryniewicz, B. E. Little, P. S. Cho, R. A. Wilson, L. G. Joneckis, and P.-T. Ho, "Wavelength conversion in GaAs micro-ring resonators," Opt. Lett. 25, 554-556 (2000).

23. L. G. Helt, Z. Yang, M. Liscidini, and J. E. Sipe, "Spontaneous fourwave mixing in microring resonators," Opt. Lett. 35, 3006-3008 (2010).

24. Y. S. Kim and M. E. Noz, Phase Space Picture of Quantum Mechanics (World Scientific, 1991). 